\title{
Longitudinal Topography and Interdigitation of Corticostriatal Projections in the Rhesus Monkey ${ }^{1}$
}

\author{
L. D. SELEMON ${ }^{2}$ AND P. S. GOLDMAN-RAKIC \\ Section of Neuroanatomy, Yale University School of Medicine, New Haven, Connecticut 06510
}

\begin{abstract}
Anterograde tracing methods were used to examine the topographic organization and interrelationship of projections to the neostriatum arising from various areas of association cortex. In contrast to the currently accepted topographic schema, all cortical areas examined project to longitudinal territories that occupy restricted medial-lateral domains of the neostriatum. The posterior parietal and superior arcuate cortices project to dorsolateral portions of the neostriatum; the dorsolateral and dorsomedial frontal cortices project centrally; and the orbitofrontal, anterior cingulate, and superior temporal projections are distributed to ventromedial regions of the caudate nucleus and putamen. In coronal section, cortical terminal fields form a diagonal strip, extending from the dorsal, ventricular border of the caudate nucleus, through the fiber bundles of the internal capsule, to the ventral margin of the putamen. Double labeling studies, in which two cortical areas were injected in the same animal, indicated that convergence of input within neostriatal domains is not governed by reciprocity of corticocortical connectivity. Thus, the interrelationship of projections arising from connectionally linked cortical areas ranged from nearly complete segregation of terminal fields (e.g., from dorsolateral prefrontal and orbital cortices) to extensive overlap of terminal domains (e.g., from frontal and temporal cortices). In the latter case, detailed analysis revealed that frontal and temporal terminals actually were interdigitated rather than intermixed within the zone of overlap. The present findings suggest a new conceptualization of corticostriatal topography in the primate which emphasizes the longitudinal arrangement of cortical terminal domains. Additionally, these findings provide a map for functional parcellation of the neostriatum on the basis of its cortical innervation which may prove useful to understanding normal striatal function, as well as the symptomatology associated with neostriatal injury and disease.
\end{abstract}

Projections to the caudate nucleus from the association cortices form part of a complex circuit through which cognitive centers influence motor output. Despite the prominence of this projection in

Received June 18, 1984; Revised August 27, 1984;

Accepted August 28, 1984

\footnotetext{
${ }^{1}$ This work was supported by United States Public Health Service Grants MH38546 and MH00298 (P. S. G.-R.) and Fellowship Award AG05310 (L. D. S.), and by a grant from the Hereditary Disease Foundation (P. S. G.-R. and L. D. S.). We wish to thank J. Treffeisen, S. Maturo, and J. Musco for excellent technical assistance.

${ }^{2}$ To whom correspondence should be addressed.
}

forebrain function, basic features of corticostriatal connectivity have not been fully resolved. The presently accepted model of corticostriatal topography is based on studies of fiber degeneration in the neostriatum following cortical lesions (Whitlock and Nauta, 1956; Devito and Smith, 1964; Johnson et al., 1968; Petras, 1969; Kemp and Powell, 1970). Seminal studies using this method describcd an anterior-posterior organization for corticostriatal terminal fields such that cortex in the frontal lobe projected to the head of the caudate nucleus, parietal cortex projected to the body, and occipital and temporal cortices projected to dorsal and ventral regions of the tail, respectively; a similar anterior posterior arrangement of cortical fields was observed in the putamen. Although this concept, i.e., that cortex projects to proximal portions of the neostriatum, is deeply entrenched in the neurological literature, it is not fully in accord with recent evidence from studies using autoradiographic pathway tracing techniques. The latter studies indicate that circumscribed areas of the association cortex project throughout all anterior-posterior (AP) subdivisions, i.e., head, body, and tail of the caudate (Goldman and Nauta, 1977b; Yeterian and Van Hoesen, 1978; Van Hoesen et al., 1981), and that the motor and premotor cortices have similar, A$P$ extensive terminal fields in the putamen (Künzle, 1975, 1977, 1978; Jones et al., 1977).

Recent anatomical tracing studies also raise a new issue regarding the convergence of projections arising from cytoarchitectonically distinct cortical areas. Yeterian and Van Hoesen (1978) and, more recently, Pearson et al. (1983) compared the projections of selected cortical regions and found that certain cortical areas that are reciprocally connected via corticocortical projections have overlapping terminal field distributions in the caudate nucleus. Although these findings have been widely interpreted to represent a general principle of organization for corticostriatal circuitry, a relatively small sample of reciprocally connected cortical areas has been examined to date. Whether such a relationship holds for all connectionally linked cortical areas remains to be determined. Moreover, analysis of convergence so far has been limited to inter-animal comparison of striatal terminal fields. With this methodology, exact determination of the degree of overlap between cortical projections is difficult since sections selected for comparison may not represent strictly analogous regions of the neostriatum.

Because uncertainties still exist concerning the relationship between the cerebral cortex and the neostriatum, further study of the basic organization of corticostriatal projections is warranted. Accordingly, the present study was undertaken to re-examine both the topographic organization and the degree of convergence or segregation in corticostriatal afferents originating from functionally distinct cortical areas. Anterograde transport methodologies have been utilized to map the corticostriatal projections of association cortices in the frontal, parietal, and temporal lobes. In addition, precise determination of the degree of convergence between cortical projections has been obtained by simultaneous labeling of two corticostriatal terminal fields in the same animal. Such information is essential for understanding the nature and neural basis of a variety 
of neurological disorders, including Huntington's chorea, that are associated with pathology of the neostriatum.

\section{Materiais and Methods}

Single labeling. Thirteen monkeys (cases 1 to 13) were injected or implanted with horseradish peroxidase (HRP) or tritiated amino acids $\left(\left[{ }^{3} \mathrm{H}\right]\right.$ $A A)$ in one of the following areas: the dorsolateral pretrontal cortex, the dorsomedial prefrontal cortex, the superior arcuate cortex, the lateral orbital cortex, the medial orbital cortex, the frontal pole, the posterior parietal cortex, or the superior temporal gyrus (Table 1).

Equal parts of tritiated proline (specific activity, $29.5 \mathrm{Ci} / \mathrm{mmol}$ ) and leucine (specific, activity, $152.0 \mathrm{Ci} / \mathrm{mmol}$ ) were evaporated and reconstituted in distilled water to a final concentration of 50 or $100 \mu \mathrm{Ci} / \mu \mathrm{l}$. HRP pellets were prepared according to the method of Griffin et al. (1979). Isotope injections and HRP implants were performed under visual guidance during sterile craniotomy.

Monkeys with injections of $\left[{ }^{3} \mathrm{H}\right]-\mathrm{AA}$ were perfused 7 to 10 days after surgery with 1 to 2 liters of phosphate-buffered saline $\left(\mathrm{pH} 7.4 ; 37^{\circ} \mathrm{C}\right), 2$ to 3 liters of $10 \%$ formalin, 2 liters of phosphate-buffered $10 \%$ sucrose $(\mathrm{pH} 7.4$; $4^{\circ} \mathrm{C}$ ), and 2 to 3 liters of phosphate-buffered $20 \%$ sucrose $\left(\mathrm{pH} 7.4 ; 4^{\circ} \mathrm{C}\right)$ The brains were embedded in gelatin, and frozen sections, cut at 40 or 50 $\mu \mathrm{m}$, were processed for autoradiographic localization of $\left[{ }^{3} \mathrm{H}\right]-\mathrm{AA}$ as described previously (Goldman and Nauta, 1977a). Monkeys receiving HRP pellet implants, injections of HRP, or injections of the lectin-conjugated enzyme wheatgerm agglutinin (WGA)-HRP were perfused following a 2-day survival period with 1 to 2 liters of phosphate-buffered saline $\left(\mathrm{pH} 7.4: 37^{\circ} \mathrm{C}\right)$ followed by 0.8 to 2.0 liters of phosphate-buffered $1.0 \%$ paraformaldehyde $+2.5 \%$ glutaraldehyde $\left(\mathrm{pH} 7.4 ; 37^{\circ} \mathrm{C}\right)$, and then 2 liters each of phosphate-buffered $10 \%$ and $20 \%$ sucrose $\left(\mathrm{pH} 7.4 ; 4^{\circ} \mathrm{C}\right)$. HRP was reacted using the tetramethylbenzidine (TMB) method of Mesulam (1978).

Double labeling. To examine the interrelationship and possible convergence of striatal projections from different cortical areas, five monkeys (cases 14 to 18 ) received double injections: $\left[{ }^{3} \mathrm{H}\right]$-AA were injected into one cortical site, and HRP pellets were implanted into a second cortical area in the same hemisphere (Table I). Large amounts of tracers were used to ensure that the entire striatal terminal field of each cortical projection was labeled and to maximize the possibility of terminal field overlap. In each of the five animals, one injection site was centered in the dorsolateral prefrontal cortex (DPC); the second tracer was placed in a cortical area that is anatomically connected with the DPC. These areas were the posterior parietal cortex (PPC), the orbitofrontal cortex (ORB), the anterior cingulate gyrus (ACG), and the

TABLE I

Amount and placement of anterograde tracers

\begin{tabular}{|c|c|c|c|c|}
\hline Case No. & Cortical Site & Brodmann's Area & Tracer & Amount \\
\hline 1 & Dorsolateral prefrontal & 9,10 & {$\left[{ }^{3} \mathrm{H}\right] \cdot \mathrm{AA}$} & $30 \mu \mathrm{Ci}$ \\
\hline 2 & Dorsolateral prefronta! & 9 & {$\left[{ }^{3} \mathrm{H}\right]-\mathrm{AA}$} & $20 \mu \mathrm{Ci}$ \\
\hline 3 & Dorsolateral prefrontal & 9,10 & {$\left[{ }^{3} \mathrm{H}\right]-\mathrm{AA}$} & $30 \mu \mathrm{Ci}$ \\
\hline 4 & Dorsomedial frontal & 9 & HRP & 5 pellets \\
\hline 5 & Dorsomedial frontal & 9 & WGA.HRP & $6 \mu$ of $2.5 \%$ \\
\hline \multirow[t]{2}{*}{6} & Dorsal premotor & 6 & {$\left[{ }^{3} \mathrm{H}\right]-\mathrm{AA}$} & $300 \mu \mathrm{Ci}$ \\
\hline & $\begin{array}{l}\text { (opposite hemi- } \\
\text { sphere: precentral) }\end{array}$ & 4 & {$\left[{ }^{3} \mathrm{H}\right]-\mathrm{AA}$} & $150 \mu \mathrm{Ci}$ \\
\hline 7 & Lateral orbitofrontal & 10 & {$\left[{ }^{3} \mathrm{H}\right]-\mathrm{AA}$} & $165 \mu \mathrm{Ci}$ \\
\hline 8 & Medial orbitofrontal & 11 & {$\left[{ }^{3} \mathrm{H}\right]-\mathrm{AA}$} & $40 \mu \mathrm{Ci}$ \\
\hline 9 & Medial orbitofrontal & 11 & {$\left[{ }^{3} \mathrm{H}\right]-\mathrm{AA}$} & $40 \mu \mathrm{Ci}$ \\
\hline 10 & Frontal pole & 12 & HRP & $8 \mu \mathrm{l}$ of $50 \%$ \\
\hline 11 & Posterior parietal & 7 & {$\left[{ }^{3} \mathrm{H}\right]-\mathrm{AA}$} & $100 \mu \mathrm{Ci}$ \\
\hline 12 & Superior temporal & 22 & {$\left[{ }^{3} \mathrm{H}\right]-\mathrm{AA}$} & $300 \mu \mathrm{Ci}$ \\
\hline 13 & Superior temporal & 22 & {$\left[{ }^{3} \mathrm{H}\right]-\mathrm{AA}$} & $210 \mu \mathrm{Ci}$ \\
\hline \multirow[t]{2}{*}{14} & Dorsolateral prefrontal & $9,10(8,6)$ & HRP & 8 pellets \\
\hline & Posterior parietal & 7 & {$\left[{ }^{3} \mathrm{H}\right]-\mathrm{AA}$} & $350 \mu \mathrm{Ci}$ \\
\hline \multirow[t]{2}{*}{15} & Dorsolateral prefrontal & 9,10 & {$\left[{ }^{3} \mathrm{H}\right]-\mathrm{AA}$} & $350 \mu \mathrm{Ci}$ \\
\hline & Posterior parietal & $7(5)$ & HRP & 7 pellets \\
\hline \multirow[t]{2}{*}{16} & Dorsolateral prefrontal & 9,10 & HRP & 4 pellets \\
\hline & Orbitofrontal & 10,11 & {$\left[{ }^{3} \mathrm{H}\right]-\mathrm{AA}$} & $280 \mu \mathrm{Ci}$ \\
\hline \multirow[t]{2}{*}{17} & Dorsolateral prefrontal & 9,10 & HRP & 3 pellets \\
\hline & Anterior cingulate & 24 & {$\left[{ }^{3} \mathrm{H}\right]-\mathrm{AA}$} & $150 \mu \mathrm{Ci}$ \\
\hline \multirow[t]{2}{*}{18} & Prefrontal-cingulate & $\begin{array}{l}9,10,12 \\
(11,24)\end{array}$ & HRP & 4 pellets \\
\hline & Superior temporal & 22 & {$\left[{ }^{3} \mathrm{H}\right]-\mathrm{AA}$} & $420 \mu \mathrm{Ci}$ \\
\hline
\end{tabular}

superior temporal gyrus (STG). In cases with combined DPC and PPC injections, tracers were introduced into the posterior two-thirds of the principal sulcal cortex since this is the region that is reciprocally connected to the PPC (Pandya et al., 1971; Mesulam et al., 1977; Leichnetz, 1980; GoldmanRakic and Schwartz, 1982; Schwartz and Goldman-Rakic, 1982, 1984). Anterior portions of the principal sulcal cortex were injected in combination with either the ACG or STG because the anterior DPC projects to the ACG and STG and receives reciprocal projections from these cortices (Pandya et al., 1971, 1981; Baleydier and Mauguiere, 1980; M. L. Schwartz and P. S.
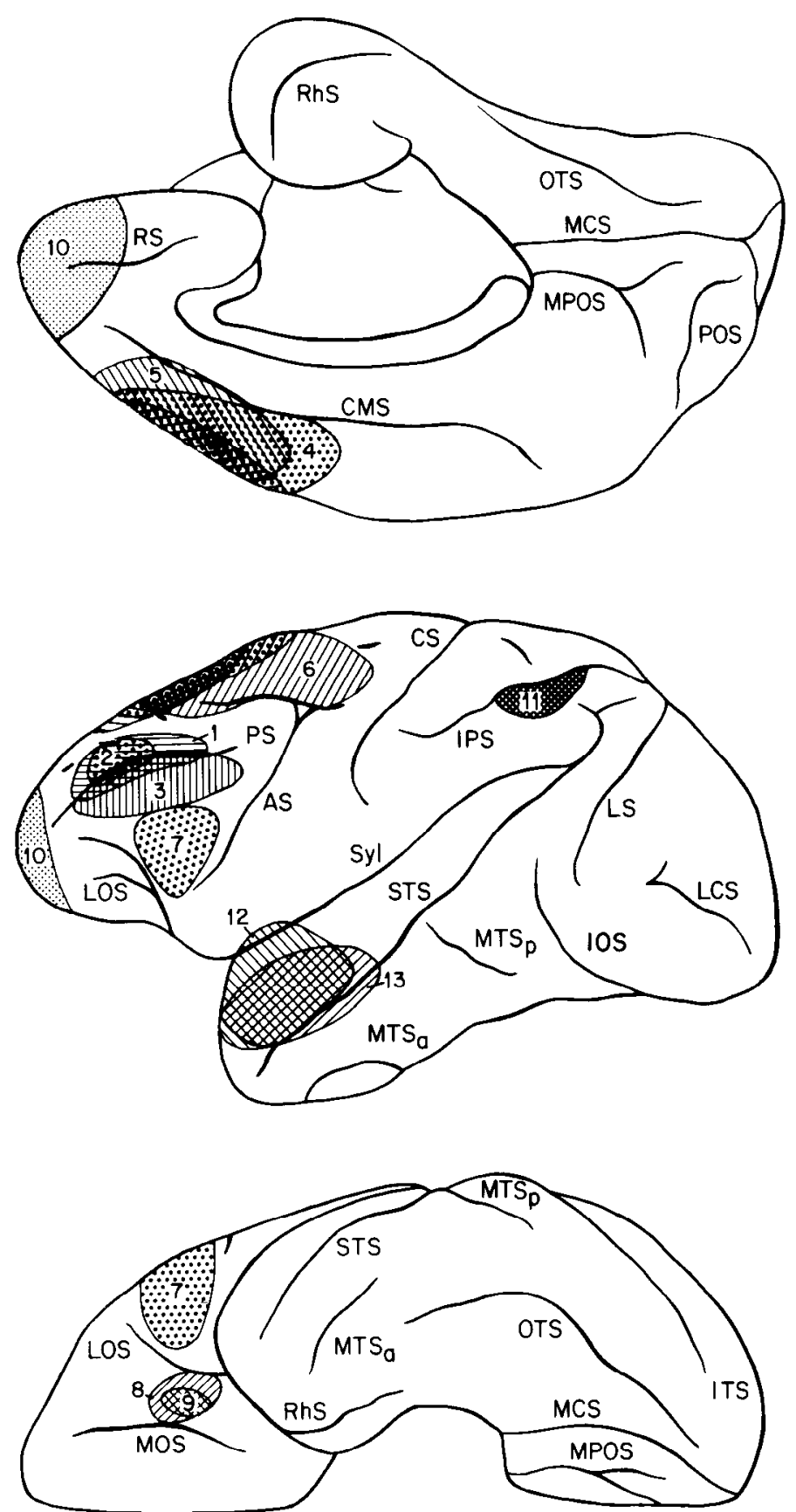

Figure 1. Injection sites of single-labeled cases (1 to 13) are shown on medial (top), lateral (middle), and ventral (bottom) views of a rhesus monkey brain. AS, arcuate sulcus; $C M S$, callosomarginal (cingulate) sulcus; $C S$, central sulcus; IOS, inferior occipital sulcus; IPS, intraparietal sulcus; ITS, inferior temporal sulcus; $L C S$, lateral calcarine sulcus; $L O S$, lateral orbital sulcus; $L S$, lunate sulcus; $M C S$, medial calcarine sulcus; MOS, medial orbital sulcus; MPOS, medial parieto-occipital sulcus; $M T S_{a}$, anterior middle temporal sulcus; $M T S_{p}$, posterior middle temporal sulcus; OTS, occipito-temporal sulcus; POS, parieto-occipital sulcus; PS, principal sulcus; $R h S$, rhinal sulcus; $R S$, rostral sulcus; Syl, Sylvian fissure; STS, superior temporal sulcus. 
Goldman-Rakic, unpublished observations). In all five cases, injection sites and the corticocortical projections between the two injected areas were mapped in order to verify reciprocal connectivity of the injected cortices.

In cases 14, 16, and 17, both anterograde tracers were administered during a single surgery. In double-labeled cases 15 and $18,\left[{ }^{3} \mathrm{H}\right]$-AA were injected 1 week before implantation of HRP pellets. All monkeys were perfused 2 days after the surgery for implanting HRP pellets. Adjacent frozen sections were processed for autoradiography and HRP histochemistry, respectively.

Although many of the cortical terminal fields examined in the present study were distributed bilaterally in the neostriatum, analysis of topography and overlap was restricted to the ipsilateral hemisphere. Ipsilateral corticostriatal projections were examined and photographed under darkfield illumination. In the double-labeled cases, selected pairs of adjacent sections were drawn using a zoom stereomicroscope equipped with a camera lucida attachment to compensate for differential shrinkage of sections during processing. These drawings were superimposed and redrawn to obtain composite maps of the two terminal fields. Both light and heavy labeling were included in the terminal field map for each cortical projection.

\section{Results}

Topography of cortical projections to the neostriatum: Singlelabeled cases

The injection sites for all single-labeled cases (1 to 13) are shown in Figure 1. While it is noteworthy that, in all single and doublelabeled cases examined in this study, terminal fields were discontinuous such that dense areas of label partially or entirely surrounded areas lacking terminal investment, this fenestrated patterning of terminal fields will not be described in detail since the major focus of the present study is the relative topography of cortical projections to the neostriatum

\section{Projections of the dorsal frontal cortex}

All three injections in the DPC bordering the principal sulcus (cases 1 to 3 ) resulted in terminal labeling throughout virtually the entire A-P extent of the caudate nucleus and also in anterior portions of the putamen (Fig. 2). Topographically, the terminal field in case 1 was notable in two respects: it occupied a central position within the nucleus and took the form of a wide band that extended diagonally from the dorsal ventricular surface of the caudate to the ventral border of the putamen (Figs. 2 and $3 A$ ). The terminal fields in cases 2 and 3 were very similar to that of case 1 and exhibited the same band-like distribution stretching from the ventricular surface to the ventral border of the striatum. However, the dorsal bank of the principal sulcus (case 2) appeared to project more laterally within the striatum relative to the projection of the ventral bank (case 3 ).

In cases 4 to 6 , the projections of cortices dorsal to the principal sulcus were examined. The projection of the dorsomedial supplementary motor cortex in cases 4 and 5 covered a large central territory of the striatum and thus resembled the projection of the dorsolateral cortex both in location and in the band-like pattern of termination (Fig. 4). Unlike the dorsolateral prefrontal terminal zone, however, the dorsomedial projection was present throughout the entire A.P extent of the putamen, including its posterior limit (Fig. 4). The neostriatal projection of the premotor cortex (case 6) terminated in lateral sectors of the head and body of the caudate and extended throughout lateral portions of the putamen (Fig. 4). At all A-P levels of the neostriatum, the terminal field of the premotor cortex exhibited a banded distribution. In this case the putaminal terminal field, in part, may reflect a crossed projection from the contralateral precentral gyrus (see Table I). Nonetheless, it is clear that the lateral striatal projection of the premotor cortex is topographically distinct from the more centrally located projection of the DPC.

\section{Projections of the ventral frontal cortex}

The striatal projections of the lateral (case 7) and medial orbitofrontal cortices (cases 8 and 9) also extended throughout the entire longitudinal extent of the caudate nucleus and into the anterior putamen but appeared to be medially displaced relative to the projection of the DPC (Figs. 2, 3, A and $B$, and 5). In cases 8 and 9 with medial orbitofrontal injections, the striatal projections were more
Figure 2. The projection of the dorsolateral prefrontal cortex (case 1) to the neostriatum is shown on standard coronal sections extending throughout approximate A-P levels A +26 to $A+8$. Terminals were located centrally in the head, body, and tail of the caudate, and in precommissural portions of the putamen. A +26 . Silver grains were present in the most anterior portion of the head of the caudate nucleus. $A+23$ and $A+20$, Likewise, at more posterior levels of the neostriatum that included both the head of the caudate and the adjacent putamen, the field of terminal labeling formed a band that extended from the dorsal surface of the caudate through the in ternal capsule, to the ventral margin of the putamen. At this level, as in the more anterior sections, a large medial territory and a smaller lateral area of the striatum did not contain terminal labeling. $A+17$ to $A+8$, Within the body and dorsal tail of the caudate, labeled terminals maintained a central position but were restricted to the dorsal portion of the nucleus adjacent to the ventricle and did not extend ventrally to reach the internal capsule. $A+11$ and $A+8$, Sparse terminal labeling was observed medially in the ventral tail of the caudate.
$A+26$
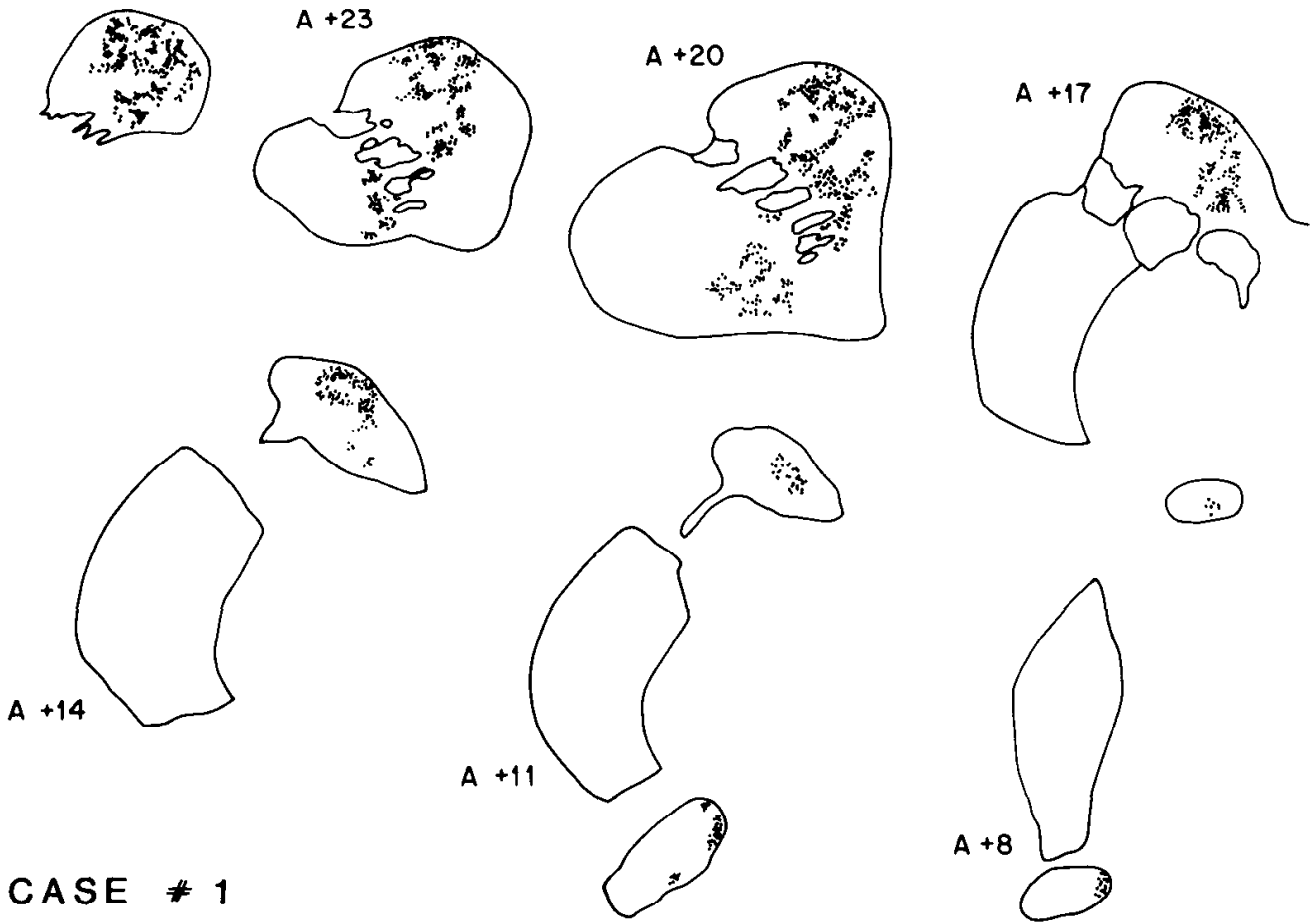

CASE $\# 1$

DORSOLATERAL PREFRONTAL CORTEX

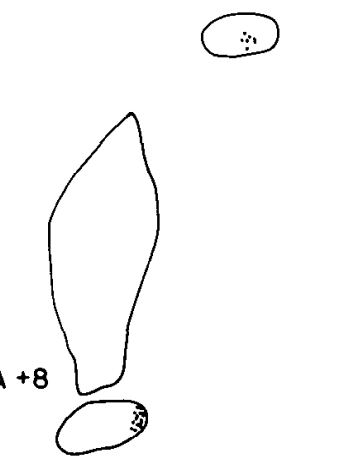



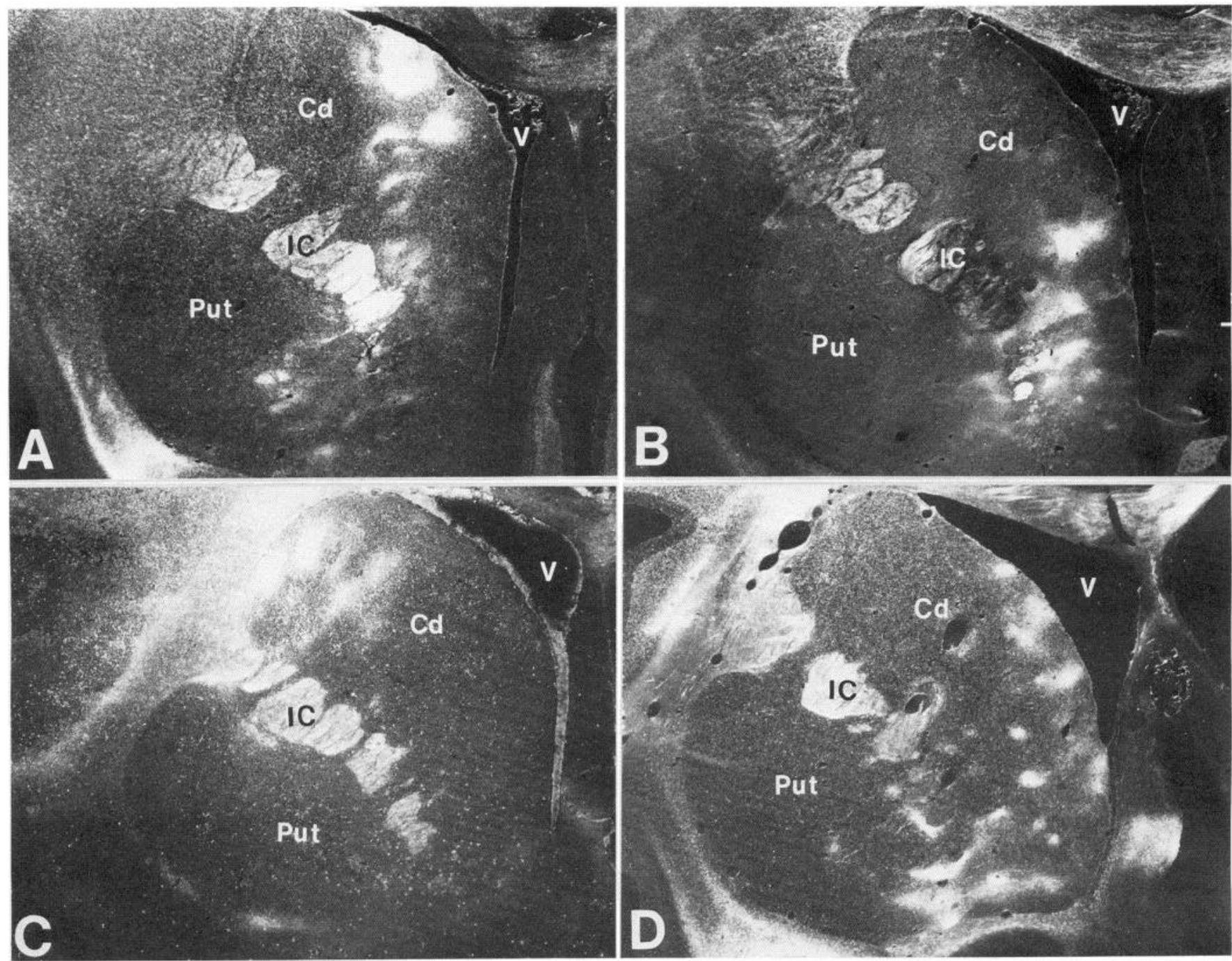

Figure 3. The topography of corticostriatal projections is illustrated in darkfield photomicrographs at approximate A-P level $\mathrm{A}+20$. $A, D$ orsolateral prefrontal (case 1), $B$, orbitofrontal (case 8), $C$, posterior parietal (case 11), and $D$, superior temporal (case 12) terminal fields are shown in comparable coronal sections through the neostriatum. Note that each cortical projection occupies a distinct medial-lateral sector of the neostriatum and extends from the dorsal border of the caudate nucleus $(C d)$ to the ventral margin of the putamen (Put). IC, internal capsule; $V$, lateral ventricle. Magnification $\times 5.8$

limited in the head of the caudate, and more extensive in mid-regions of the putamen, than those of the lateral orbitofrontal cortex (case 7).

Following implantation of HRP pellets in the frontal pole (case 10), a projection was observed throughout the head, body, and dorsal tail of the caudate nucleus. However, in any given coronal section, only a few dense patches of label were present within a small territory that corresponded to the most lateral portion of the orbitofrontal projection.

\section{Projections of the posterior parietal cortex}

The projection of the PPC extended throughout a longitudinal territory of the neostriatum that included the head, body, and dorsal tail of the caudate, as well as anterior and posterior regions of the putamen (Fig. 6). In the precommissural neostriatum, the appearance was that of a band spanning the lateral neostriatum from the dorsolateral margin of the head of the caudate to the ventrolateral border of the putamen (Figs. $3 \mathrm{C}$ and 6 ). In the body of the caudate nucleus, the terminal field was situated at the dorsolateral border of the nucleus but shifted position in the dorsal tail where it bordered the ventral margin of the caudate at a distance from the lateral wall. A few patches of terminal labeling were situated ventrally and somewhat laterally within the posterior putamen (Fig. 6).

\section{Projections of the superior temporal gyrus}

The striatal projections in cases 12 and 13 were identical. Terminal labeling was observed throughout all A-P levels of the caudate and in the anterior putamen (Fig. 7). In the head of the caudate, labeled terminals covered roughly the ventromedial third of the nucleus and were in contiguity with terminal labeling in the anterior putamen (Figs. $3 D$ and 7). Projections were distributed medially within the body of the caudate; in both dorsal and ventral tail regions, a band of labeled terminals was visible just medial to the center of the caudate (Fig. 7).

\section{Interrelationship of terminal domains in the striatum: Double- labeled cases}

\section{Topographic relationship of dorsolateral prefrontal and posterior} parietal terminal fields

Injection sites. The frontal injection site in case 14 included both dorsal and ventral banks of the posterior principal sulcus and the adjacent dorsal convexity of the frontal lobe (Figs. 8 and 9). HRP spread into the dorsal bank of the superior arcuate sulcus and into the cortex posterior to the principal sulcus. The parietal injection involved the rostral two-thirds of the posterior bank of the intraparietal sulcus and adjacent convexity (Figs. 8 and 9). In case 15, the frontal 
Figure 4. The neostriatal projection of the dorsomedial frontal cortex (supplementary motor area) is shown above (case 5). $A+26$ and $A+23$, At anterior levels of the neostriatum, the terminal field nearly filled the head of the caudate and adjacent putamen. $A+20$, More posteriorly in the precommissural neostriatum, terminal labeling formed a dorsoventrally oriented band in central regions of the caudate and putamen. $A$ +17 to $A+8$, This band-like pattern of termination was evident more posteriorly as well, where label was present centrally in the body and dorsal tail of the caudate and medially in subjacent regions of the posterior putamen. The projection of the dorsal bank of the superior arcuate sulcus (premotor area) is represented below (case 6). $A+26$ to $A+20$, Terminals were located in lateral portions of the head of the caudate and anterior putamen. $A+17$ and $A+14$, Only a small ventrolateral territory in the body of the caudate contained label, whereas the projection extended throughout a large lateral territory of the underlying, medial putamen. $A+11$ and $A+8$, in the posterior neostriatum, terminal labeling was observed in dorsolateral regions of the putamen but not in either dorsal or ventral portions of the tail of the caudate.
$\mathrm{A}+26$
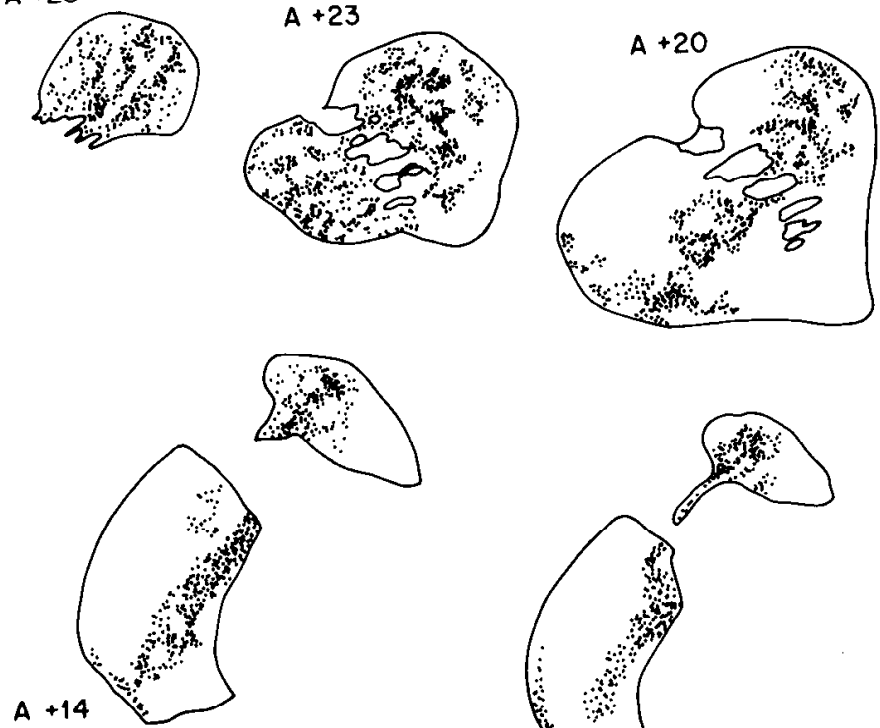

CASE \#5
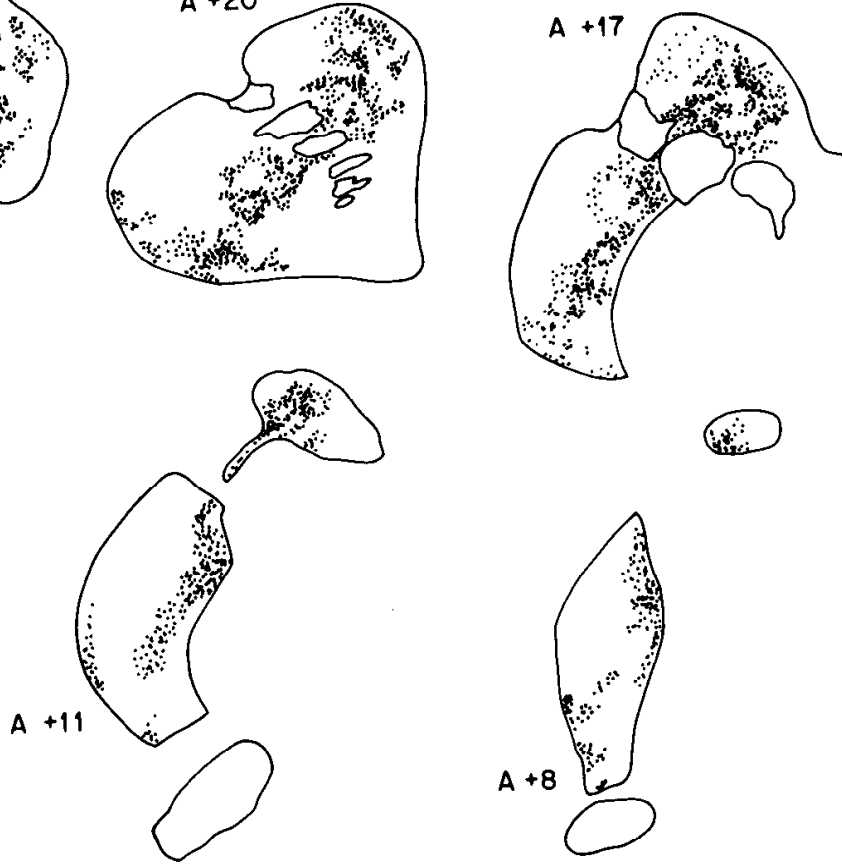

DORSOMEDIAL FRONTAL CORTEX
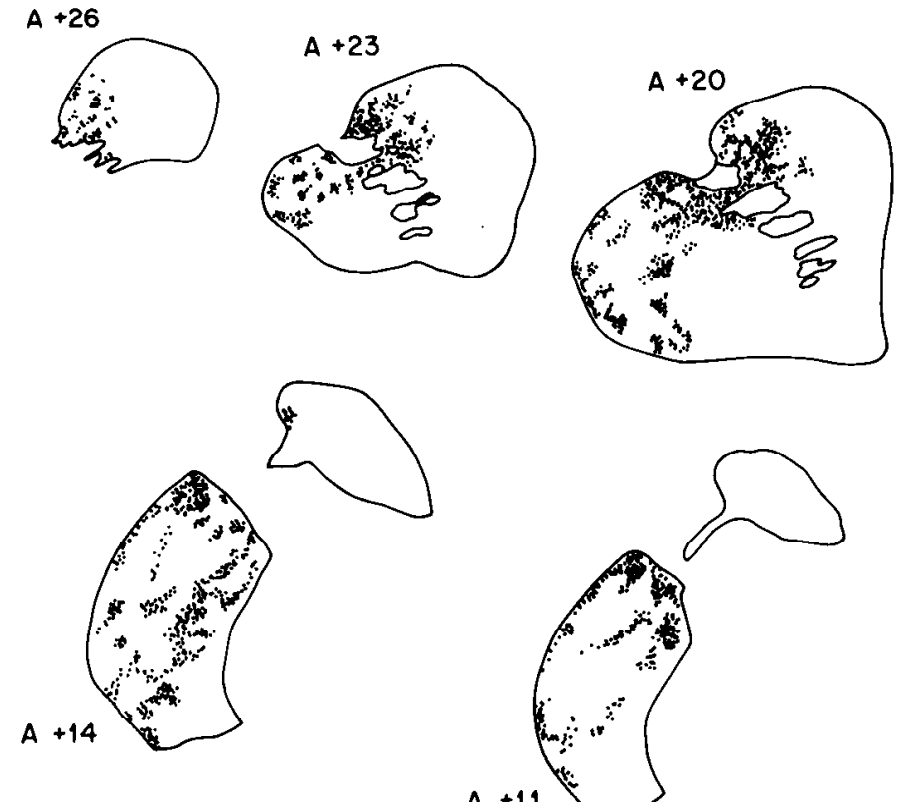

$A+11$
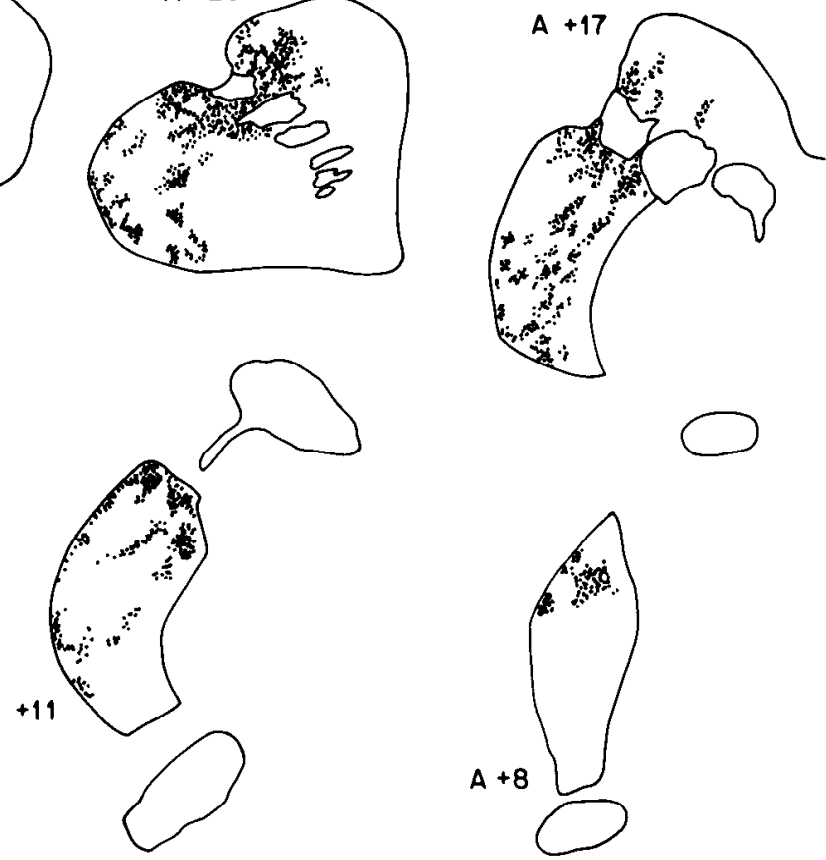

CASE \#6

\section{SUPERIOR ARCUATE CORTEX}

injection sitc was smaller than that of case 14 and was confined largely to the posterior two-thirds of the dorsal bank of the principal sulcus; however, the parietal injection site in case 15 was larger than the corresponding injection site in case 14, involving both anterior and posterior banks of the intraparietal sulcus as well as the cortex dorsal to the posterior extension of the superior temporal sulcus in the parietal lobe. Comparison of the frontal and parietal injection sites with respective corticocortical projection zones revealed that 
$A+26$
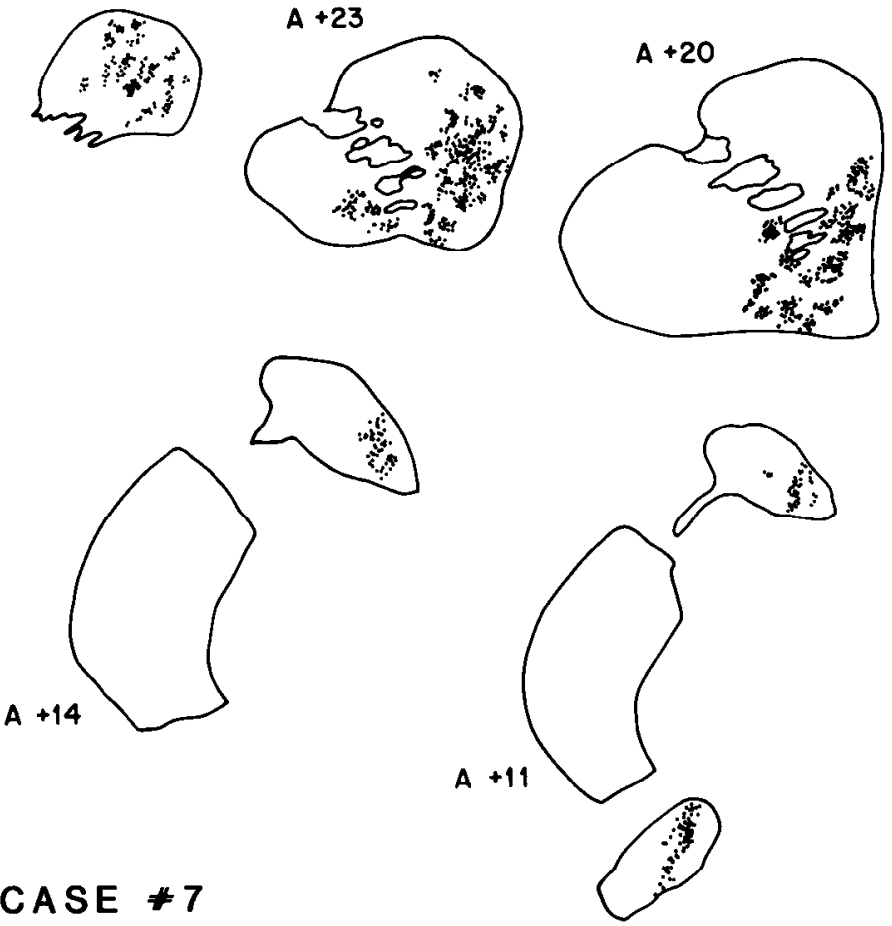

CASE $\# 7$

ORBITOFRONTAL CORTEX

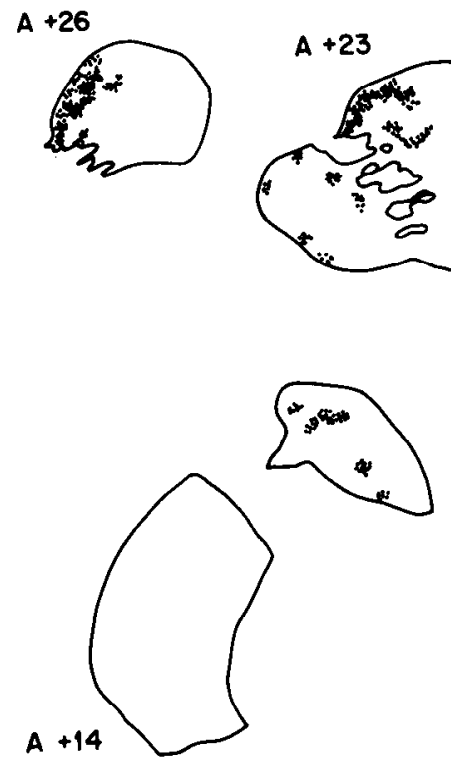

A +11
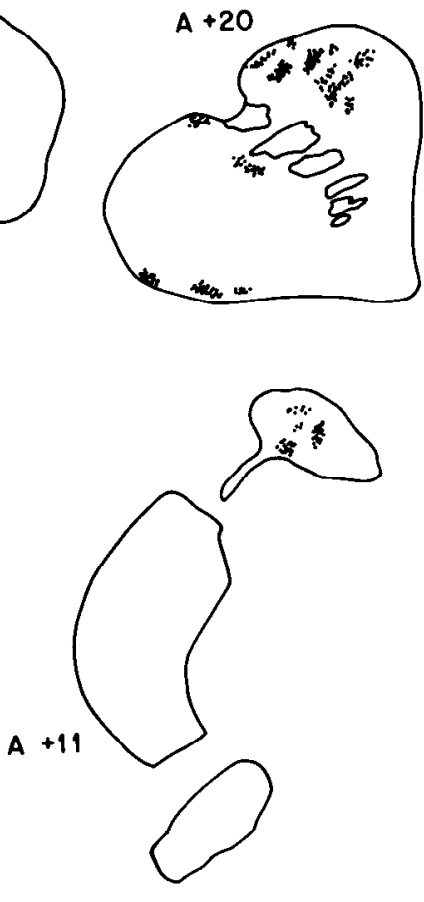

CASE * 11

\section{POSTERIOR PARIETAL CORTEX}

the injected cortices represented reciprocally connected cortical areas (for details, see Fig. 8).

Corticostriatal projections. As expected from the results of single injections in these two cortical regions, the PPC terminal field was situated laterally in the striatum relative to the DPC terminal field (Figs. 9 and 10). Where the DPC projection was most extensive

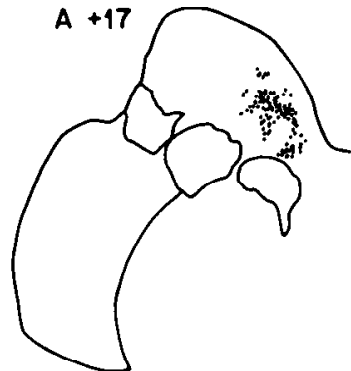

Figure 5. The projection of the lateral orbitofrontal cortex (case 7) to the neostriatum is shown on standard sections. $A+26$, In the most anterior portion of the caudate nucleus, terminals filled a wide central area that appeared to be medially located relative to the projection of the dorsolateral cortex. $A+23$ and $A+20$. At more posterior levels of the striatum, the terminal field formed a wide band extending from the dorsomedial ventricular surface of the caudate, through the internal capsule, to the ventromedial limit of the precommissural putamen. $A+17$ to $A+8$, Labeled terminals also were present in the body and dorsal tail of the caudate in approximately the same ventromedial position observed in the head of the caudate. $A+11$, In the ventral tail, terminal labeling occupied a narrow, dorsoventrally oriented strip located a short distance from the medial wall of the nucleus.
Figure 6. The neostriatal projection of the posterior parietal cortex (case 11) is shown on standard sections. $A+26$ to $A+20$, Terminal labeling was located laterally in the anterior caudate and putamen. $A$ +17 to $A+8$, In the body and dorsal tail of the caudate, the posterior parietal projection shifted to occupy a central sector of the nucleus. $A+8$, Patches of terminal labeling also were observed in ventral regions of the posterior putamen. (i.e., in the head of the caudate), the PPC terminal field was small, and, conversely, where the PPC terminal field was largest (i.e., in the body of the caudate), the DPC projection was diminished in size. Overlap of DPC and PPC terminal fields was restricted to the mutual boundary of these adjacent domains and was prominent only at far anterior levels of the head of the caudate (Fig. 9). 
A +26

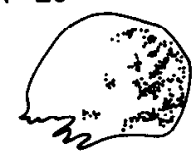

$A+23$
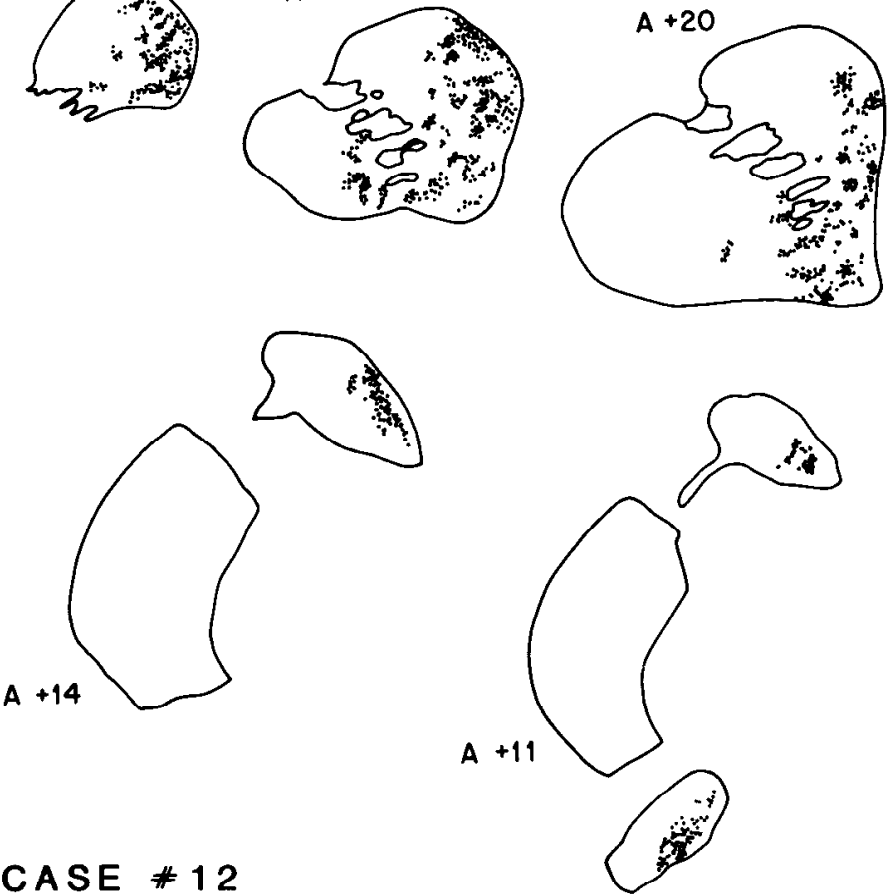

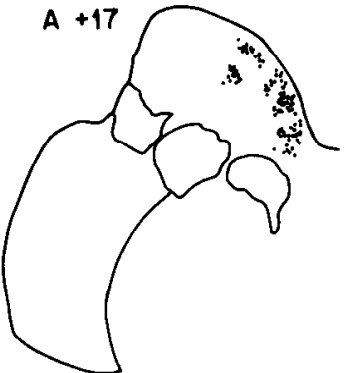

.. neostriatum, the terminal zone included ventromedial portions of the head of the caudate and adjacent, medial regions of the putamen. $A$ +17 and $A+14$. Label was observed medially in the body of the caudate. $A+11$ and $A+8$, Terminals also were observed in dorsal and ventral regions of the tail.

Figure 7. The neostriatal termina(case 12) is illustrated on standard terminals were distributed over a broad ventromedial territory of the nucleus. $A+23$ and $A+20$, More
CASE *12

\section{SUPERIOR TEMPORAL GYRUS}

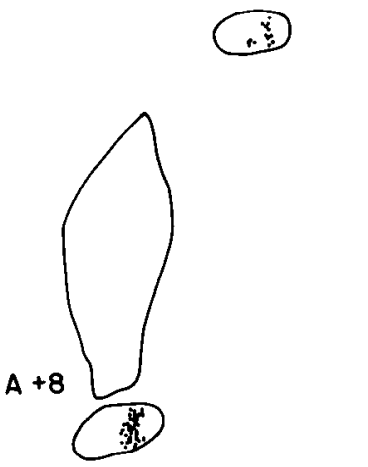

Topographic relationship of the dorsolateral prefrontal and orbitofrontal terminal fields

Injection sites. In case 16 the HRP injection site extended in an A-P direction to encompass nearly all cortex surrounding the principal sulcus (Fig. 11). Although the injection site did not extend to the medial surface of the hemisphere, it did spread through the white matter to involve the cortex overlying the fundus of the callosomarginal (cingulate) sulcus. At rostral levels, the $\left[{ }^{3} \mathrm{H}\right]-\mathrm{AA}$ injection site included the lateral convexity of the frontal lobe extending from the ventral border of the principal sulcal cortex to the medial orbital sulcus (Fig. 11). Mid-regions of the injection site included the gyrus rectus and medial orbital cortex, whereas at caudal levels of the frontal lobe, it was restricted to the orbital cortex underlying the striatum. The HRP and $\left[{ }^{3} \mathrm{H}\right]-\mathrm{AA}$ injection sites overlapped very slightly on the lateral convexity of the frontal lobe (Fig. 11). Analysis of corticocortical projections between the orbital and dorsolateral cortices confirmed that the injected cortices were reciprocally connected, although the orbital projection to the dorsolateral convexity appeared to be more robust than the reverse projection.

Corticostriatal projections. For the most part, the ORB projection was located ventral and medial to the DPC terminal field throughout the precommissural neostriatum, although some overlap of ORB and DPC terminal label was observed in the head of the caudate and anterior putamen (Figs. 11 and $12, A$ and $B$ ). In the body and dorsal tail of the caudate, a portion of the DPC terminal field overlapped ORB labeling in the ventromedial sector, but much of the DPC terminal field was located outside the ORB projection in the central sector of the nucleus (Fig. 11). The DPC and ORB projection reversed positions in the ventral tail such that ORB terminals were situated medial to DPC terminals (Fig. 11).

Topographic relationship of the dorsolateral prefrontal and anterior cingulate terminal fields

Injection sites. In case 17 the HRP injection site involved the principal sulcal cortex, with the exception of the most anterior portion of the ventral bank (Fig. 13). HRP spread caudally and ventrally from the injection site to include the cortex ventral and posterior to the principal sulcus and a small lateral portion of the rostral head of the caudate nucleus. In addition, HRP spread through the white matter underlying the principal sulcus and into the cortex subjacent to the callosomarginal (cingulate) sulcus. The radiolabeled site was largely confined to the anterior cingulate cortex with slight involvement of the contralateral ACG and of the dorsomedial cortex on the medial wall of the hemisphere (Fig. 13). Although a prominent dorsolateralcingulate projection was observed, a return projection was not found in autoradiograms of the DPC. However, neurons in the ACG were retrogradely labeled from the HRP injection site in this case (case 17 ) and in other cases with HRP in the DPC (cases 14 and 16).

Corticostriatal projections. At anterior levels of the striatum, the spread of HRP from the DPC injection site seemed to block the reaction of the DPC terminal field. As a result, only sparse DPC terminal labeling was observed surrounding the injection site in the anterior striatum (Fig. 13). Therefore, direct determination of the degree of overlap between the DPC and ACG terminal zones in the anterior striatum was not possible. However, comparison of the ACG terminal field in case 17 with the DPC projection zone from other single- and double-labeled cases suggests that these cortical projections probably overlap in the anterior putamen but not in the head of the caudate where the DPC terminal field is located centrally and the ACG projection terminates ventromedially. Throughout the rest of the neostriatum, the DPC projection was located centrally and therefore was separated from the ventromedial termination of the ACG projection (Figs. 12, $C$ and $D$, and 13).

It should be noted that the topography of the ACG terminal field reported here differs from that of previous studies which localized the anterior cingulate projection more laterally within the neostriatum (Yeterian and Van Hoesen, 1978; Baleydier and Mauguiere, 1980). At present, we have no explanation for this discrepancy.

\section{Topographic relationship of prefrontal-cingulate and superior temporal terminal fields}

Injection sites. The HRP injection site in case 18 covered more than the intended anterior two-thirds of the dorsolateral cortex surrounding the principal sulcus. HRP saturated the lateral orbital cortex, as well as adjacent dorsomedial and lateral convexities of 


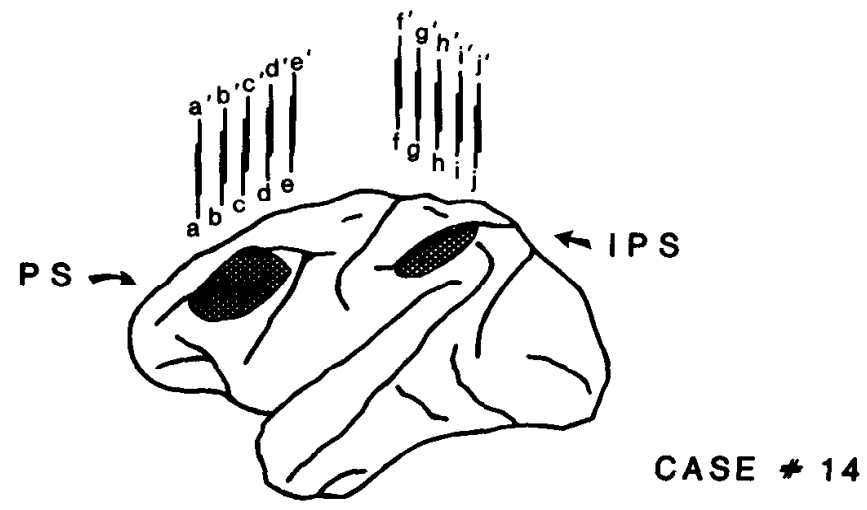

INJECTION SITES

PROJECTION ZONES

H R P

a

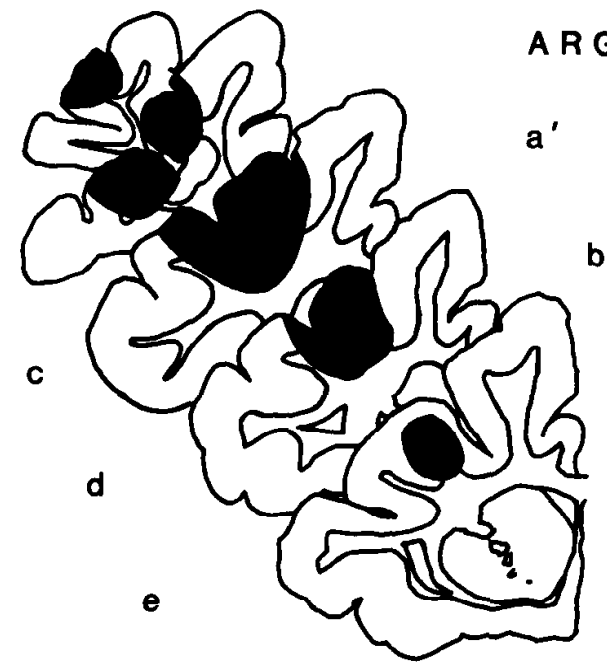

A R G

$b^{\prime}$
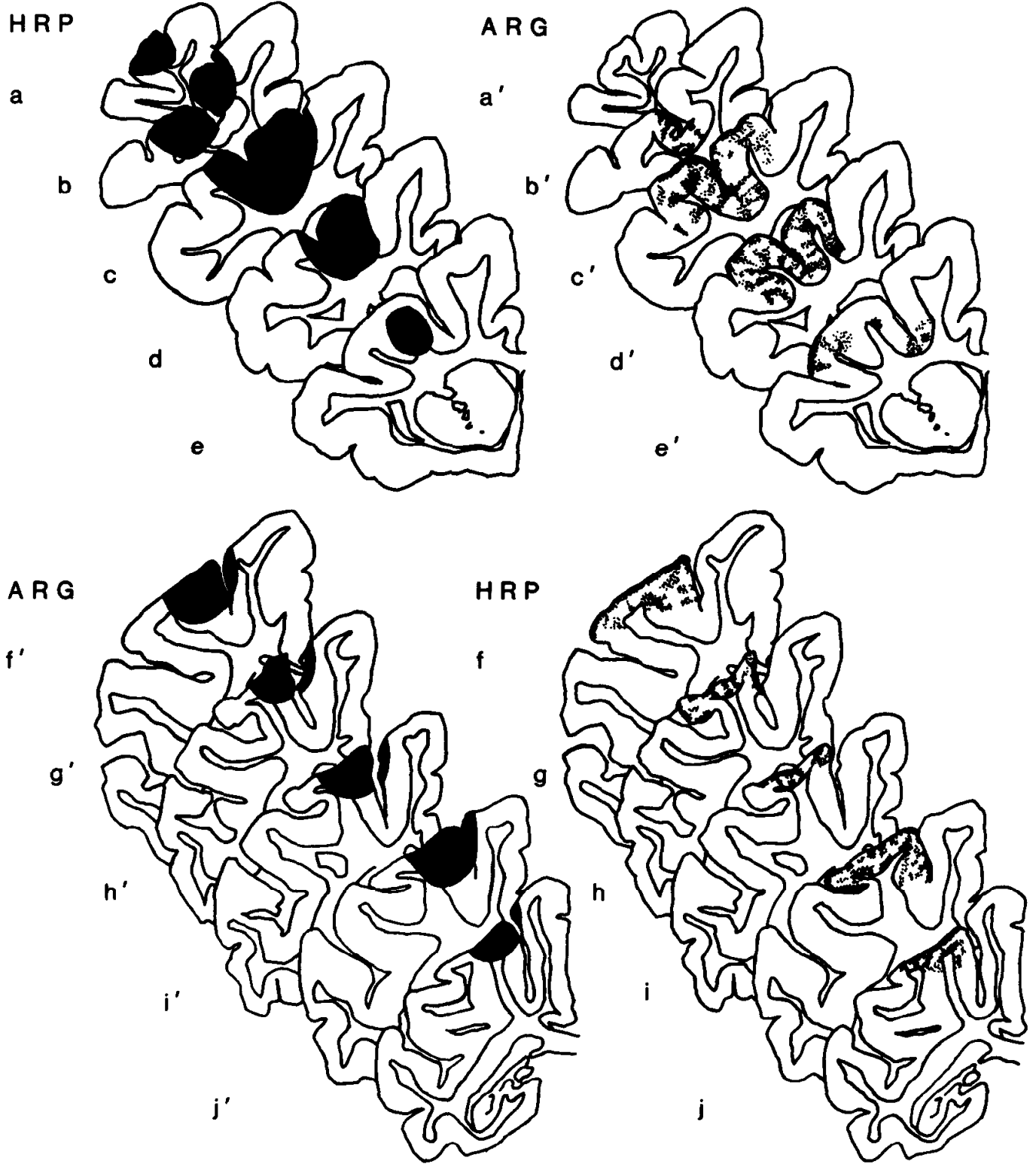

Figure 8. Drawings of adjacent coronal sections, processed for HRP histochemistry ( $a$ to $j)$ and $\left[{ }^{3} H\right]$-AA autoradiography $(A R G)\left(a^{\prime}\right.$ to $\left.j^{\prime}\right)$, respectively, illustrate reciprocal, corticocortical connectivity between dorsolateral prefrontal and posterior parietal injection sites in case 14. The HRP-saturated injection site $\left(a\right.$ to $e$ ) in the frontal lobe closely matches the projection zone of corticocortical afferents $\left(a^{\prime}\right.$ to $\left.e^{\prime}\right)$ originating from the parietal injection site. Likewise, mapping of the dorsolateral prefrontal projection to the posterior parietal lobe revealed a close correspondence between the termination of prefrontal afferents $(f$ to $j)$ and the $\left[^{3} \mathrm{H}\right]-A A$ injection site in the parietal lobe $\left(f^{\prime}\right.$ to $\left.j^{\prime}\right)$. PS, principal sulcus; IPS, intraparietal sulcus. 

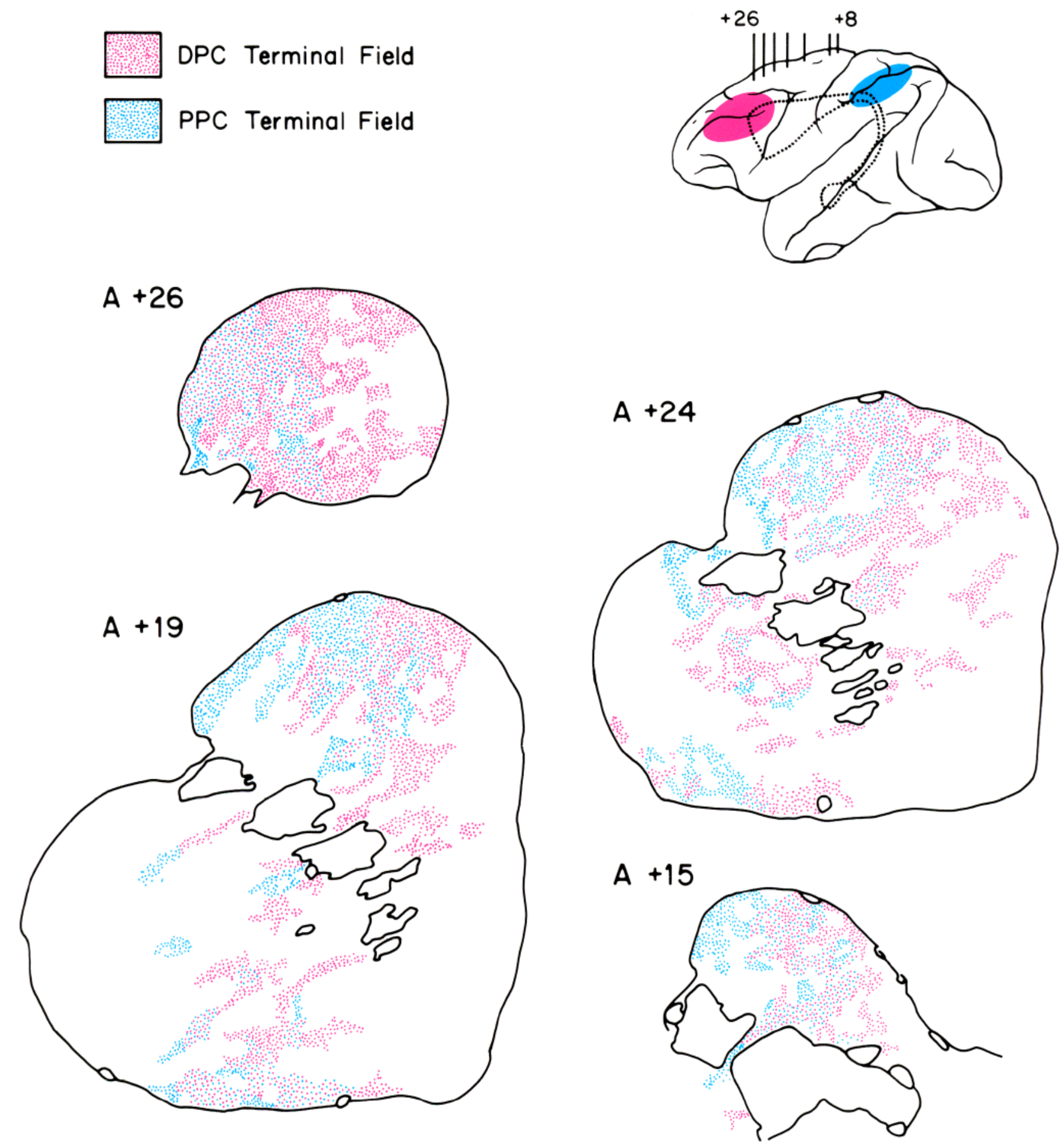

$A+10$
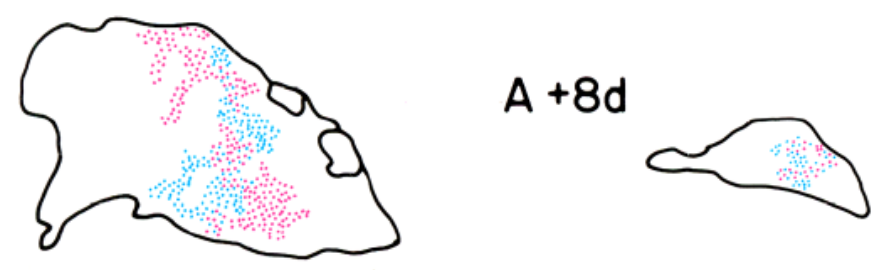

\section{$A+8 v$}


the frontal lobe, and spread through the white matter to infiltrate the fundus of the callosomarginal (cingulate) sulcus and the cortex dorsal and rostral to this sulcus (Fig. 14). The $\left[{ }^{3} \mathrm{H}\right]-A A$ injection site was confined to the temporal pole and the cortex on the dorsal bank of the STG (Fig. 14). Since the frontal injection site included several cytoarchitectonic areas, it will be referred to as "prefrontal-cingulate (P-C)." Reciprocal connections linking the frontal and temporal injection sites were present: P-C terminals were found in the temporal pole area and along the dorsal bank and convexity of the STG; projections from the STG were observed on the dorsomedial and lateral convexities of the frontal lobe and in the depths of the callosomarginal (cingulate) sulcus.

Corticostriatal projections. In the precommissural neostriatum, P. $C$ terminals were distributed to a broad, band-like territory that included both central and ventromedial sectors of the head of the caudate and adjacent putamen (Fig. 14). At this level the STG terminal field filled the ventromedial sector and encroached upon central regions of the neostriatum as well (Fig. 14). Given the topographic similarity in the two terminal domains, one might have expected considerable overlap of terminal labeling. However, such overlap was actually remarkably limited. Analysis of adjacent sections reacted for visualization of HRP and processed for autoradiography, respectively, allowed precise determination of the interrelationship of the P-C and STG fields. A striking finding was that, throughout the overlapping zone, the terminal fields were interdigitated so that patches of P-C terminal labeling fit into the empty spaces in the STG terminal field and vice versa, thus forming a fine mosaic pattern (Figs. 12, $E$ and $F$, and 14). In selected sections where the interdigitation was pronounced, measurement of the cross-sectional width of several adjacent but discrete terminal zones showed them to be variable but in the range of 350 to $900 \mu \mathrm{m}$. More posteriorly in the neostriatum, the P-C and STG terminal fields segregated into distinct spatial zones: $P$-C terminals occupied central regions of the body and dorsal tail of the caudate, whereas STG terminal labeling was located more medially. However, overlap of $P$ $C$ and STG terminal fields was observed in the ventral tail of the caudate.

Based on the data from single-labeled cases, we attribute the topographic convergence in this case largely to overlap between the temporal lobe projection and those of the cingulate and lateral ORBs, but we cannot rule out some contribution from the anterior principal sulcal cortex. Comparison of cases with DPC injection sites centered in the anterior principal sulcus (cases 16 and 17) with those centered more posteriorly (cases 14 and 15) indicates that, relative to the posterior $\mathrm{DPC}$, the anterior DPC projects farther ventromedially in the neostriatum.

\section{Discussion}

Medial-lateral organization of corticostriatal terminal domains. Corticostriatal projections of the association cortices examined in the present study terminated in parasagittally extensive, longitudinal domains within the striatum that extended throughout all A-P subdi- visions of the caudate and included portions of the anterior putamen as well. As illustrated in Figure 15, each elongated cortical projection occupied a restricted medial-lateral sector of the striatum: the posterior parietal terminal field extended throughout dorsolateral sectors of the striatum, the dorsolateral prefrontal terminal field occupied central regions of the caudate and rostral putamen, and the orbitofrontal, superior temporal, and anterior cingulate projections to the striatum were located ventromedially. This newly defined mediallateral organization for corticostriatal input differs substantially from the widely accepted A-P topography that has been described for cortical projections to the striatum (Whitlock and Nauta, 1956; Devito and Smith, 1964; Johnson et al., 1968; Petras, 1969; Kemp and Powell, 1970). The discrepancy between the A.P organizational schema, which is based on fiber degeneration studies, and the present results may be explained by the superior sensitivity of the anterograde transport methodologies. In all probability, early studies which relied on silver impregnation of degenerating corticostriatal terminals detected only the largest or densest portion of each terminal field. For instance, only the projection to the head of the caudate from the DPC was recognized prior to the advent of amino acid autoradiography. Recent studies have shown that circumscribed areas of cortex project not only to proximal regions of the neostriatum, i.e., anterior cortex to anterior striatum and posterior cortex to posterior striatum, but also extend over long A-P distances to invade non-proximal portions of the neostriatum (Goldman and Nauta, 1977b; Yeterian and Van Hoesen, 1978; Van Hoesen et al., 1981). The present, more comprehensive study establishes that many cortical areas project to parasagittally elongated domains and therefore suggests that the longitudinal pattern of termination represents a generalized feature of most or all corticostriatal afferents.

Previous studies have described a medial-lateral topography for subsets of cortical input to the striatum. For instance, Kemp and Powell (1970) found that cortical areas within a single lobe projected onto the caudate nucleus as a continuous and overlapping mediallateral array. More recently, a medial-lateral organization has been described for the somatotopic representation of the body in the projection of the motor and sensory cortices onto the putamen (Künzle, 1975, 1977, 1978; Jones et al., 1977). The medial-lateral topography described in the present study differs from that of earlier studies in that projections from the association cortices do not represent a point-to-point transfer of cortical topographical relationships since nonadjacent (e.g., prefrontal and parietal) cortices project to adjacent neostriatal territories. We propose that the segregation and alignment of projections from the association cortices into medial-lateral domains instead reflects an underlying functional parcellation of the neostriatum. It is relevant, therefore, that recent studies of striatal connectivity in the rat have led to the hypothesis that the nucleus accumbens represents a ventromedial, limbic extension of the striatum and, therefore, that the rat striatum is functionally differentiated into neocortically receptive (dorsolateral) and allocortically receptive (ventromedial) divisions (Kelley and Domesick, 1982; Kelley et al., 1982; Haber and Nauta, 1983), although

Figure 9. A composite map showing the locations of the PPC projection, labeled with $\left[{ }^{3} \mathrm{H}\right]-\mathrm{AA}$, and the DPC terminal field labeled with HRP, in the neostriatum in case 14. The approximate A-P plane of each composite drawing is indicated. $A+26$, At anteriormost levels, PPC.terminals (blue) were restricted to the dorsolateral quadrant, while DPC terminals (red) covered all but a ventromedial wedge of the nucleus. Thus, there was considerable overlap of the two projection fields at this level, but more than half of the DPC field was situated outside the boundary of the PPC terminal field. $A+24$ and $A+19$ More posteriorly, but still within the head of the caudate, the PPC projection maintained its relative size and position dorsolaterally while the DPC terminal field narrowed to occupy a dorsomedial, juxtaventricular region of the nucleus. Because the DPC terminal field was much smaller here than in more anterior sections, the zone of overlap between DPC and PPC projection fields also diminished in size. In the anterior putamen, the DPC terminal field was medially displaced relative to the smaller PPC field, although some overlap of the DPC and PPC zones was apparent. $A+15$, Within the body of the caudate, the PPC projection filled the dorsolateral quadrant and also encroached upon the dorsomedial area of the caudate adjacent to the lateral ventricle, whereas the DPC terminal field continued to decrease in size and to regress farther from the lateral border of the nucleus. As a result, only a slender band of overlap was present at the common boundary of the two cortical terminal fields. $A+10$ and $A+8$, in the dorsal tail of the caudate, the PPC projection was located more centrally within the nucleus but maintained its lateral position with respect to the DPC field. Although terminal distributions of both DPC and PPC were sparse in the ventral portion of the tail, PPC and DPC terminal fields exhibited the same lateral and medial topography, respectively, that had been evident in dorsal and anterior divisions of the caudate. 

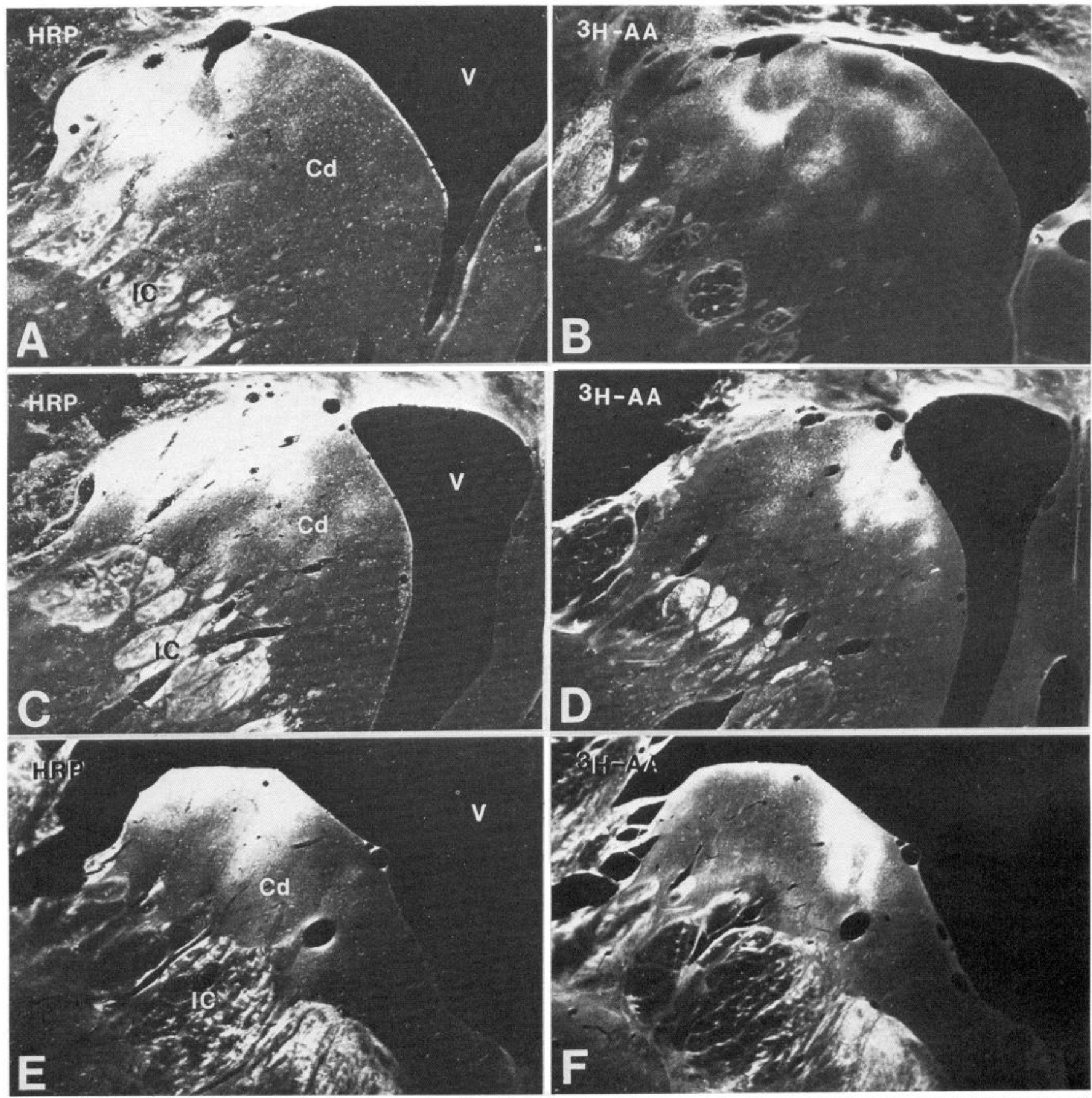

Figure 10. Darkfield photomicrographs of adjacent coronal sections illustrating the relative topography of projections from the PPC and DPC in case 15 . $A$ and $B$, The PPC terminal field $(A)$, which was labeled with HRP in this case, was situated laterally in the head of the caudate nucleus $(C d)$, whereas the DPC projection $(B)$, shown in adjacent autoradiograms, occupied a central position in the nucleus $(A+24)$. $C$ and $D$, At more posterior levels of the head of the caudate $(C)$, the PPC terminal domain was located laterally and was adjacent to the central termination $(D)$ of DPC afferents $(A+19)$. $E$ and $F$, In the body of the caudate, PPC and DPC projections maintained lateral and central positions within the caudate $(A+15)$. Labeled cortical fiber bundles were present within the internal capsule $(I C)$ and should not be confused with neostriatal terminal field labeling. The lateral ventricle $(V)$ is indicated for orientation. Magnification: $B, D$, and $F, \times 8.3 ; A, C$, and $E$, photographically enlarged to match $B, D$, and $F$.

Figure 11. Illustration of the relative topography and overlap of the HRP-labeled DPC projection and the radiolabeled ORB terminal field in the neostriatum (case 16). $A+25$, The DPC terminal field (red) occupied a large, centrally located territory anteriorly within the head of the caudate. At this level, ORB terminals (blue) were scattered over the ventromedial third of the caudate with very few terminals observed dorsally. $A+23$, At mid-regions of the head, the ORB projection occupied a crescent-shaped territory that protruded dorsally into the DPC terminal zone but also extended ventrally beyond the DPC terminal field to the ventromedial border of the caudate so that about half of the ORB terminal field overlapped the DPC projection zone. $A+19$, More posteriorly within the precommissural striatum, the medial-lateral topography of the ORB and DPC projections was more prominent. DPC terminals filled central territories of the head of the caudate and underlying putamen; ORB terminals were situated more medially in both striatal nuclei. Although the terminal fields overlapped, the densest portion of the DPC terminal field was located dorsally in the caudate and therefore was separated from the ORB field. $A+17$ to $A+10$, In the body and dorsal tail region, ORB terminals were medially located in comparison to the DPC terminal field. $A+10$, in the ventral tail, this topographic relationship was reversed: DPC terminals were located at the medial border of the nucleus and ORB terminals were laterally adjacent to the DPC terminal field, with little overlap apparent between the two projections. 
DPC Terminal Field ORB Terminal Field
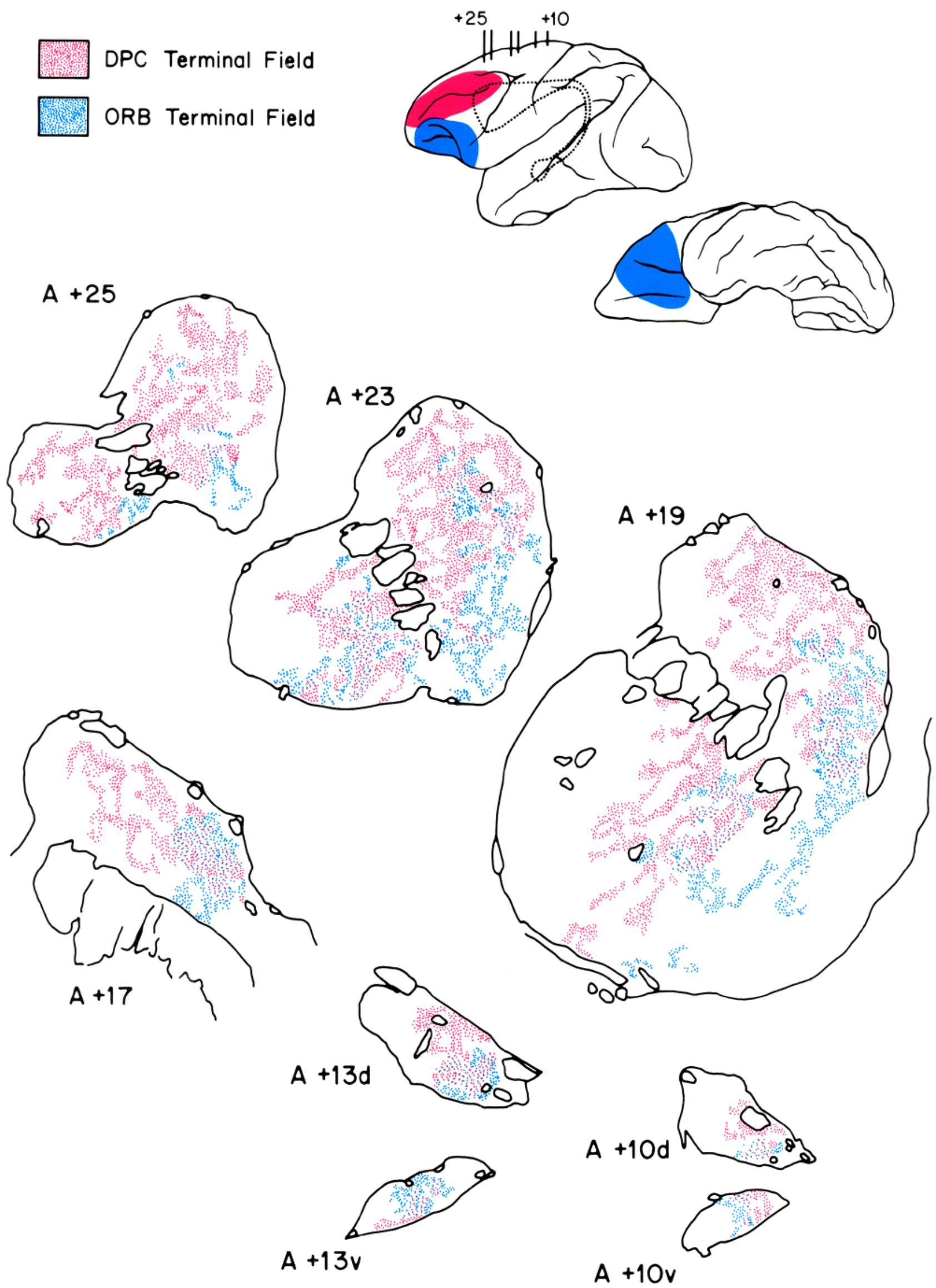

Figure 11 

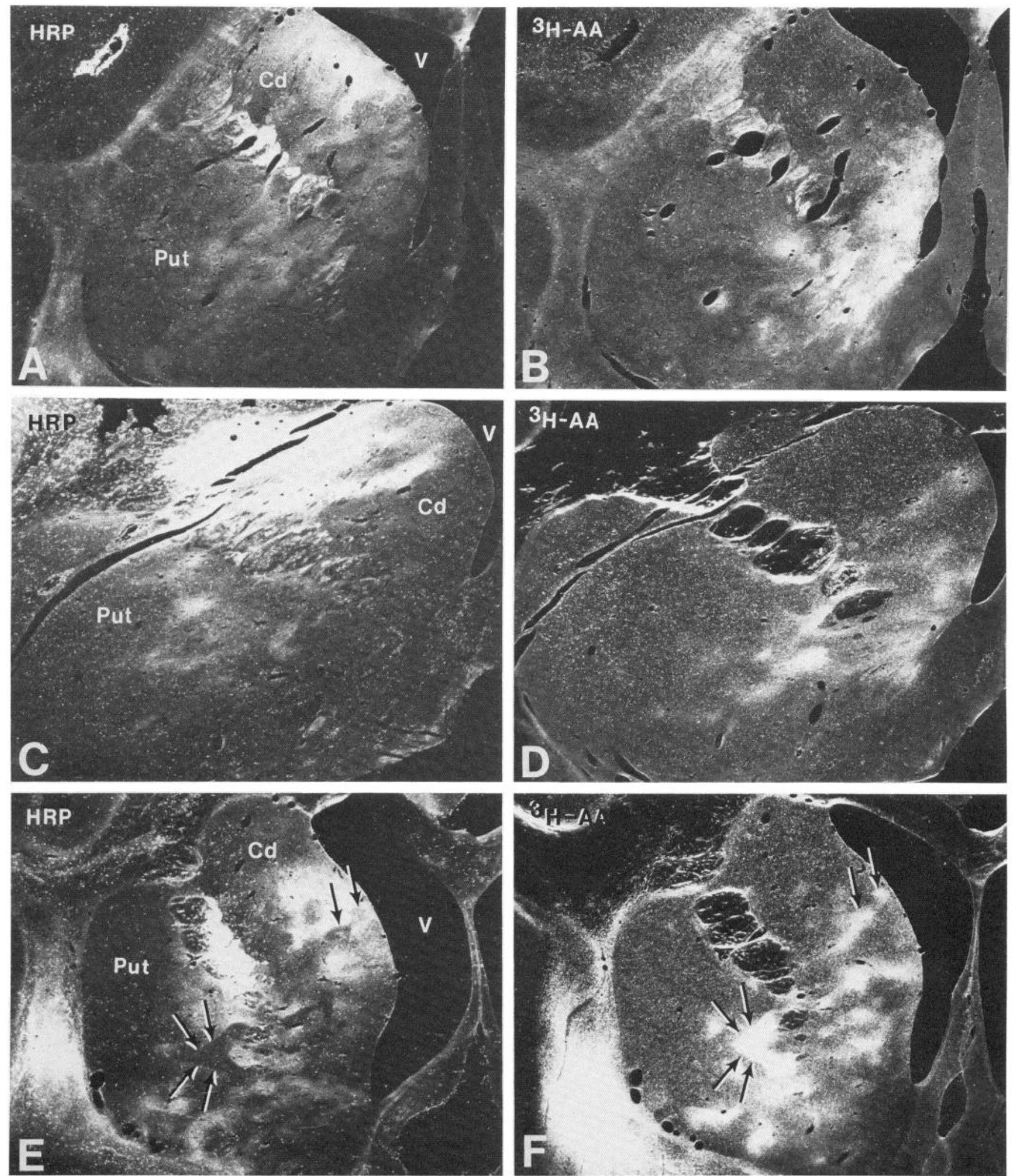

Figure 12. Darkfield photomicrographs of representative adjacent sections from double-labeled cases 16 to 18 . The HRP-labeled projection of the DPC $(A)$ terminated centrally in both the caudate $(C d)$ and putamen $(P u t)$, whereas the ORB projection zone $(B)$ was located ventromedially in an adjacent autoradiogram $(A+19)$. The DPC projection $(C)$, demonstrated with HRP histochemistry, was located centrally in the neostriatum, while in an adjacent section $(D)$, the $\left[{ }^{3} \mathrm{H}\right]$-AA-labeled projection of the ACG occupied a band-like area of the ventromedial caudate and putamen $(A+19)$. $E$ and $F$, Comparison of the terminal zone originating from a large HRP injection site that included prefrontal and cingulate cortices with the [ $\left.{ }^{3} \mathrm{H}\right]-\mathrm{AA}$-labeled projection of the STG illustrates the topographic similarity and interdigitation of these cortical fields in the neostriatum. Arrows in $E$ point to unlabeled territories that were surrounded by HRP-reactive terminals of the P-C projection, and arrows in $F$ point to corresponding $\left[{ }^{3} \mathrm{H}\right]$-AA-labeled territories in the superior temporal projection (A +20 ). Note that the sections illustrated here are not the same as those forming the composite drawing at $A+20$ in Figure 14 . IC, internal capsule; $V$, lateral ventricle. Magnification: $B, D$, and $F, \times 5.8 ; A, C$, and $E$, photographically enlarged to match $B, D$, and $F$. 
DPC Terminal Field

ACG Terminal Field
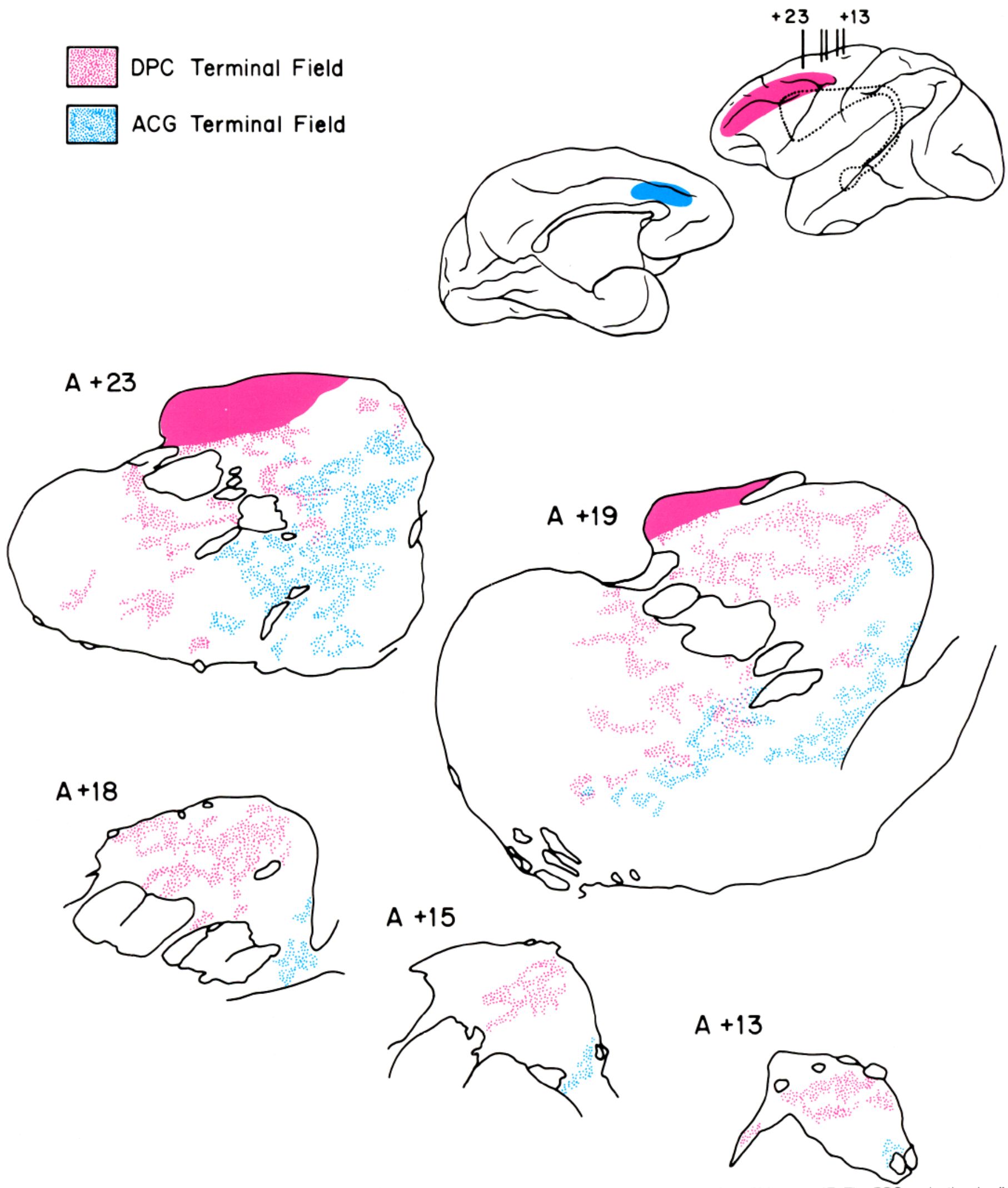

Figure 13. Superimposed drawings of the DPC and ACG projections at approximate A-P levels $(A+23$ to $A+13)$ in case 17. The DPC projection (red) was labeled with HRP; the ACG terminal field (blue) was labeled with $\left[{ }^{3} \mathrm{H}\right]-\mathrm{AA}$. $A+23$, Note that the prefrontal injection site (solid red) extended into lateral portions of the head of the caudate. DPC terminal labeling was sparse at this level and was restricted to the central neostriatum. The ACG terminal field was located ventromedially. $A+19$. Farther posteriorly in the head of the caudate and anterior putamen, DPC terminals filled a wide central area of the striatum, and ACG terminals extended throughout the ventromedial caudate and medial putamen. Superimposition of the DPC and ACG terminal fields revealed that these projections occupied adjacent, nonoverlapping territories in the caudate. In the putamen, although the projections were largely distinct, some patches of DPC terminal labeling were surrounded by terminals of the ACG projection at the mutual boundary of the two terminal zones. $A+18$, ACG terminals were restricted to a small ventromedial territory in the body of the caudate nucleus and, thus, were spatially separated from the centrally located DPC terminal domain. $A+15$ and $A+13$, In the dorsal tail region, the ACG projection again was located medially with respect to the DPC termination in the central caudate. Both DPC and ACG terminals were observed in medial portions of the ventral tail. However, adjacent sections were not available for analysis of the degree of overlap between DPC and ACG projections in this region. 

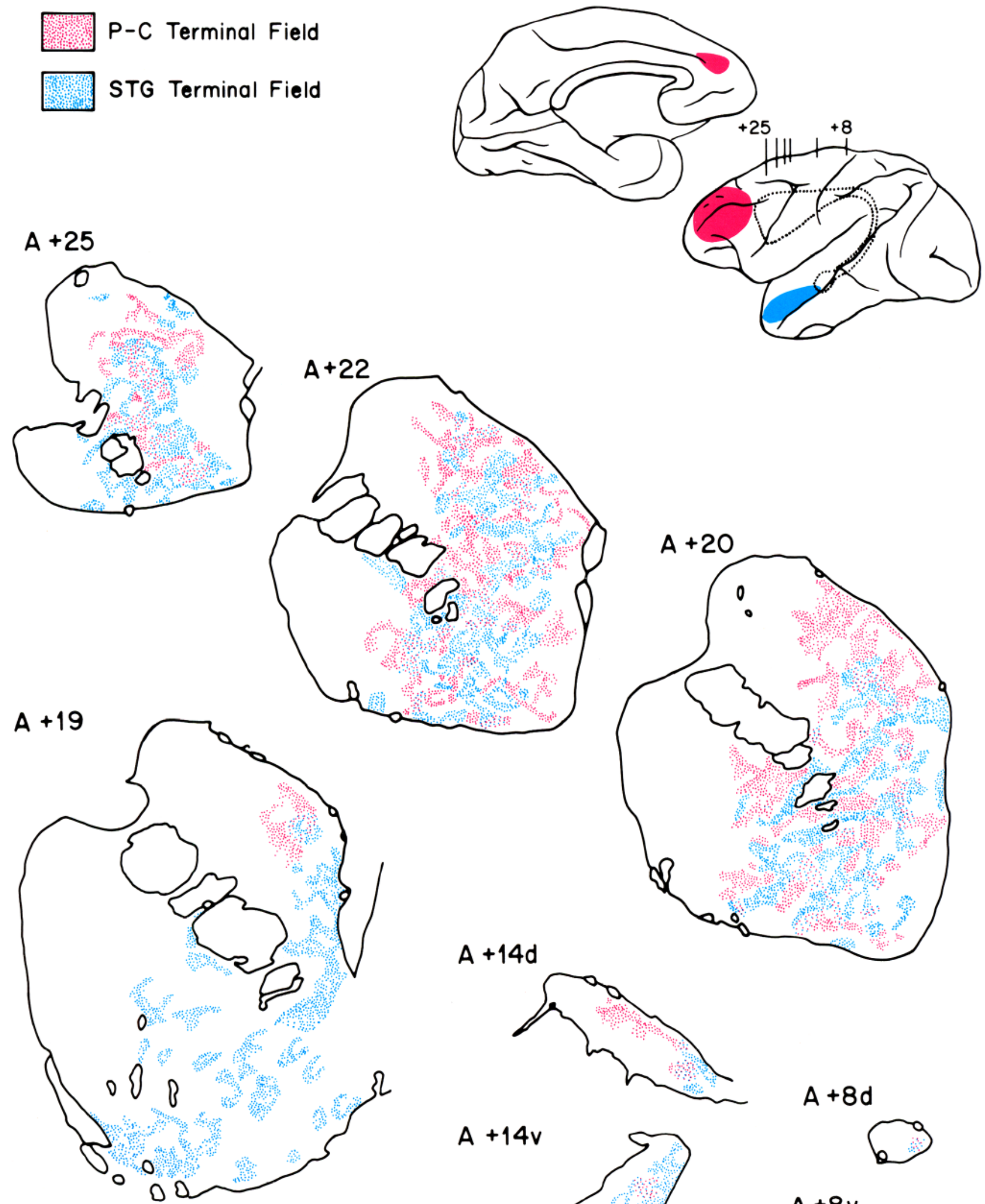

$A+14 d$
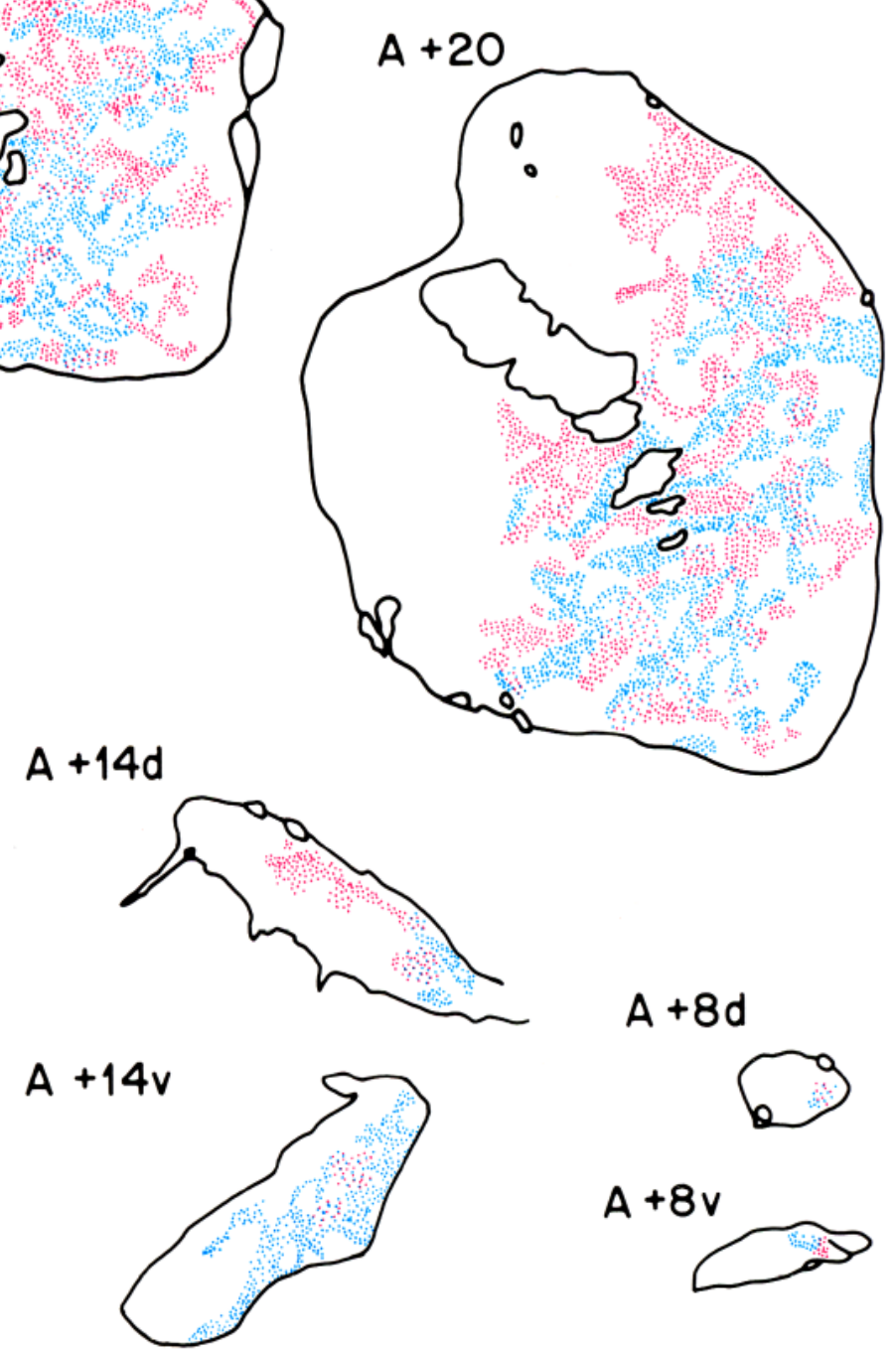

$A+8 d$

Figure 14

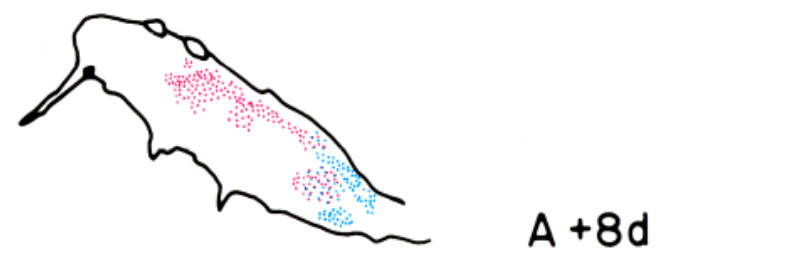




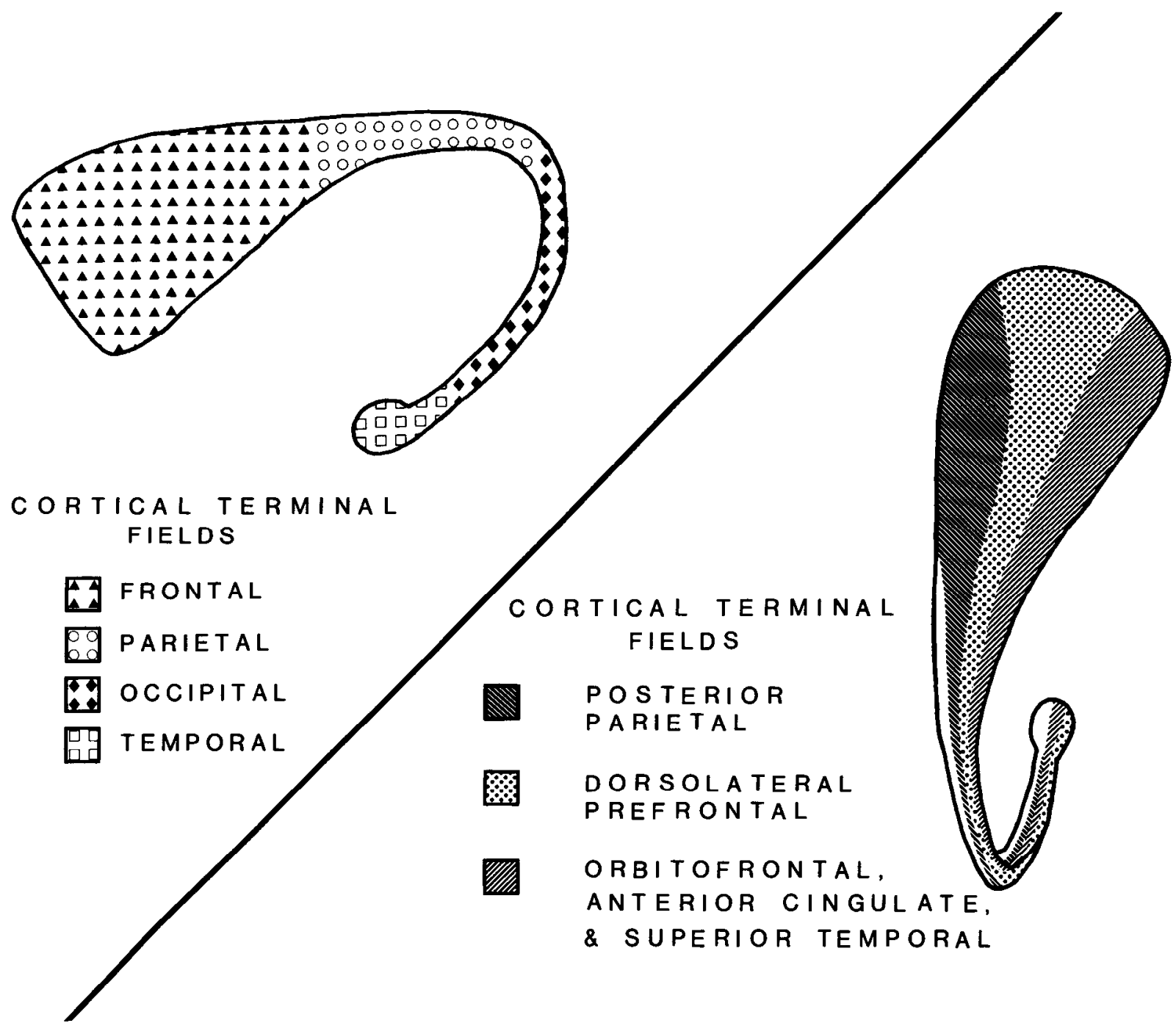

Figure 15. Comparison of the present findings to the most widely accepted model of corticostriatal topography. Upper left, A schematic representation, based on data from Kemp and Powell (1970), shows an A-P organization of cortical afferents in the caudate nucleus. Kemp and Powell's (1970) analysis of fiber degeneration in the neostriatum and several similar, although less comprehensive, studies (Whitlock and Nauta, 1956; Devito and Smith, 1964; Johnson et al., 1968; Petras, 1969) suggested that cortex in the frontal lobe projected to the head of the caudate nucleus, whereas parietal cortex projected to the body of the nucleus, and occipital and temporal cortices ramified in dorsal and ventral regions of the tail, respectively. Lower right, Results of the present study indicate that cortical afferents from the association cortices project to longitudinally extensive (i.e., spanning all A-P subdivisions) domains that are aligned along the medial-lateral axis of the caudate. Posterior parietal cortex projects dorsolaterally in the caudate; dorsolateral prefrontal cortical input terminates centrally, whereas orbitofrontal, superior temporal, and anterior cingulate projections are located ventromedially in the nucleus.

the segregation of these cortical inputs is incomplete (Beckstead, 1979). The present findings suggest that, at least in the rhesus monkey, the neocortically receptive striatum may be further differentiated into functional domains, each of which receives a unique subset of cortical projections. Whether this represents an evolutionary specialization of the primate, perhaps to accommodate the immense functional diversity of the cortex in this species, remains to be determined. However, there is some evidence from lesiontransplantation studies to suggest that similar functional domains may exist in the rat (Dunnett et al., 1981a, b).

Corticostriatal terminal domains bear a striking resemblance to the longitudinal bands described for thalamocortical projection neu-

Figure 14. A composite map of the P-C and STG projections to the neostriatum constructed by superimposition of drawings from adjacent sections ( $A$ +25 to $A+8)$ in case 18. Terminals originating from a frontal HRP injection site $(P-C)$ that involved several prefrontal areas are shown in red; the radiolabeled STG terminal field is shown in blue. A +25, P-C terminals occupied a large territory in the anterior head of the caudate; only dorsolateral and ventromedial margins of the nucleus were free of P-C terminal labeling. The smaller STG terminal field overlapped the P-C projection extensively, although lateral portions of the P-C field were located outside the STG terminal domain. $A+22$ and $A+20$, At more posterior levels, both STG and P-C projections covered roughly two-thirds of the head of the caudate and anterior putamen with only a small dorsal region of the P-C projection located outside the STG terminal field. A large portion of the P-C projection therefore overlapped the STG terminal field. However, within this zone of topographic overlap the terminal fields were interdigitated such that intermixing of P.C and STG terminals was very limited. $A+19$, Near the juncture of the head and body of the caudate, the terminal fields segregated into distinct spatial zones such that the STG field was located medially with respect to the P-C dorriain. The STG terminal field extended farther posteriorly in the putamen than did the P-C projection to occupy medial portions of the putamen. $A+14$ and $A+8$, in the postcommissural striatum, the P.C and STG terminal fields were found in nonoverlapping, central and medial regions, respectively, of the dorsal tail of the caudate. However, terminal field overlap was observed in the ventral tail where both projections were located medially. 
rons in the rhesus monkey (Kievit and Kuypers, 1977). Although the full significance of longitudinal organization in both corticostriatal and thalamocortical circuits remains to be determined, the presence of a medial-lateral topography in two distinct telencephalic systems may indicate that this topographic arrangement is related to a fundamental developmental mechanism or anatomic property of the central nervous system.

Segregation of corticostriatal projections. A double labeling strategy has been utilized in the present study to test the hypothesis that reciprocally connected cortices project to overlapping terminal zones in the striatum. Examination of adjacent sections displaying projections from the DPC and from connectionally linked cortices, respecitvely, has demonstrated that overlap is not the preponderant organizational arrangement of these terminal fields. The present findings do not represent an exhaustive examination of corticostriatal connectivity and, therefore, cannot rule out the possibility that other pairs of cortically linked striatal afferents have genuinely overlapping terminal projections. Nonetheless, the present results do indicate that overlap of cortical terminal fields cannot be predicted on the basis of corticocortical connectivity. Therefore, although selected reciprocally connected areas of cortex have been shown to project to overlapping regions of the caudate (Yeterian and Van Hoesen, 1978; Pearson et al., 1983), this connectional arrangement does not represent a general principle of organization. Rather, convergence of input in the corticostriatal system represents functional interaction between distant cortical centers that can be determined only empirically at this time.

It should be noted that some overlap of cortical terminal fields in the neostriatum was observed at the mutual boundary of adjacent prefrontal and parietal terminal domains, particularly in the anteriormost head of the caudate. Without ultrastructural data it will remain inconclusive whether this overlap is artifactual, resulting from the inclusion of fibers of passage in the composite maps of paired terminal fields. Since the anterograde transport methodologies utilized in the present study label axons as well as terminals, one could argue, for instance, that a part of the dorsolateral prefrontal projection to the head of the caudate could be due to labeling of fibers passing from the subcallosal fasciculus through the posterior parietal terminal domain to ramify centrally within the nucleus. However, it is unlikely that fibers of passage constitute a significant part of neostriatal labeling in the present study. Corticostriatal axons are fine caliber in diameter and, perhaps because of this, axon-like processes were very rarely observed in the caudate or putamen. Moreover, there is evidence from Golgi studies to suggest that corticocaudate axons are draped with boutons en passage such that these axons make synaptic contact with striatal neurons along their entire length (DiFiglia et al., 1978). Therefore, it is our view that the vast majority of neostriatal labeling in the present study consisted of terminals and boutons en passage. On this basis, we conclude that convergence of prefrontal and parietal cortical input, although limited, does occur in the neostriatum and may be more pronounced in the head of the caudate than at more posterior levels. Nonetheless, the most salient feature of the interrelationship of the prefrontal and parietal projections is the relative medial-lateral distribution of these terminal fields. Overall, the termination of prefrontal and parietal terminal domains is remarkably distinct in spite of the fact that these areas are richly interconnected via corticocortical connections (Leichnetz, 1980; Goldman-Rakic and Schwartz, 1982; Schwartz and Goldman-Rakic, 1982, 1984).

Interdigitation of corticostriatal terminal fields. In the present study, substantial topographical overlap was observed in the striatal projections of the frontal and temporal cortices, but detailed analysis of the interrelationship between these projections revealed that the frontal and temporal fields were actually interdigitated, rather than superimposed, in the area of topographic overlap. It is possible that dendrites of the medium spiny neuron, which are known to receive cortical synapses (Kemp and Powell, 1971; Kocsis et al., 1977; Hassler et al., 1978) and to extend over distances of $200 \mu \mathrm{m}$ (Pasik et al., 1976) could be contacted by both of the spatially alternating terminal areas. However, given the width of the interdigitating terminal areas (350 to $900 \mu \mathrm{m}$ ), many neurons that are contacted by one cortical input undoubtedly are not synaptically contacted by the interdigitated cortical input and vice versa. Moreover, one recent model of neostriatal organization suggests that medium spiny neurons form a lateral inhibitory matrix such that the excitation generated by spatial convergence of input is enhanced and sharpened by inhibition of neurons surrounding the focal point of excitation (Groves, 1983). Therefore, this model is consistent with the idea that interdigitated terminal fields would produce spatially distinct centers of excitation that in turn would selectively activate distinct subsets of neostriatal neurons. Of interest in this regard is that fact that the caudate nucleus may be divided into "island" and "matrix" compartments on the basis of cell density and clustering (Goldman-Rakic, 1982). Recently, the dorsolateral prefrontal projection to the caudate has been shown to terminate preferentially in the matrix compartment and to avoid the cellular islands (Goldman-Rakic, 1982), whereas preliminary analysis of the relationship between the superior temporal projection and these cytologically defined compartments indicates that the superior temporal cortex projects to both island and matrix compartments (L. D. Selemon and P. S. Goldman-Rakic, unpublished observation).

In the present study, interdigitation of cortical terminal fields was observed in the ventromedial part of the neostriatum. In addition to the marked interdigitation of frontal and temporal fields, this interlocking arrangement of terminal territories also was observed to a limited extent at the intersection of the dorsolateral prefrontal field with adjacent orbital and cingulate fields in the ventromedial neostriatum. Whether cortical afferents that converge on dorsolateral and central sectors also interdigitate remains to be determined by additional double-labeling studies. In the present study prefrontal and parietal terminals appeared to be truly intermixed, not interdigitated, in regions where the two fields overlapped and were often observed to surround the same unlabeled territory, presumably an island. These observations suggest that the hodological organization of the ventromedial sector may differ from that of the rest of the neostriatum. In addition, there is some indication in the literature that certain histochemically defined compartments exhibit mirror-image distributions in dorsolateral and ventromedial parts of the caudate nucleus. For example, Ragsdale and Graybiel (1981) found that patches low in acetylcholinesterase reactivity corresponded to labeled terminal areas of the frontostriatal projection in the dorsolateral caudate of the cat, whereas in ventromedial portions of the nucleus, acetylcholinesterase-rich areas were observed within the frontostriatal terminal field. Likewise, in the dorsolateral caudate nucleus, enkephalin-rich and substance $P$-poor patches were aligned, whereas in the ventromedial caudate nucleus, enkephalin-rich and substance P-rich patches were sometimes found in register with each other (Graybiel et al., 1981).

Finally, it is noteworthy that the interdigitation of corticostriatal terminals is reminiscent of the interdigitation of columnar corticocortical labeling in the association cortices (Goldman-Rakic and Schwartz, 1982) and of ocular dominance columns in the visual cortex (Hubel et al., 1974). Development of the striatal and cortical interdigitation appears to be similar as well. The ontogenetic transformation of a diffuse prefrontostriatal projection to an intricately patterned terminal field (Goldman-Rakic, 1981) resembles the developmental sequence leading to laminar segregation of retinal terminal fields in the lateral geniculate nucleus and to formation of ocular dominance stripes in striate cortex (Rakic, 1976). Since segregation of right and left retinal projections in the lateral geniculate nucleus is dependent on competition between these terminal fields (Rakic, 1981), analogous competition between P-C and temporal cortical afferents may account for the interdigitation of these fields in the neostriatum. Although the functional significance of spatially alternating terminal fields is not altogether clear, one may speculate that interdigitation represents a generalized anatomic mechanism 
for processing information from two or more cortical sources in the central nervous system.

Functional heterogeneity of the striatum. Over the past decade anatomical studies in primates have established that cortical input to the caudate nucleus originates largely from the association cortices, whereas the putamen receives cortical projections from sensory, motor, and premotor cortices (Künzle, 1975, 1977, 1978; Goldman and Nauta, 1977b; Yeterian and Van Hoesen, 1978; Van Hoesen et al., 1981). Moreover, recent evidence suggests that the caudate and putamen have distinct efferent targets; that is, virtually the entire projection of the caudate terminates in the substantia nigra, whereas the putamen projects almost exclusively to the globus pallidus (Parent et al., 1984). Few studies of neostriatal physiology have been carried out in the monkey, but those that have bcen done support the concept of a "division of labor" in the striatum such that the caudate is involved preferentially in cognitive processing, while the putamen subserves primarily motor function. With singleunit recording in the head of the caudate nucleus, Niki et al. (1972) have identified neurons that are selectively activated during performance of the delayed response task, a task long associated with the DPC (Goldman and Rosvold, 1970; Goldman et al., 1971), whereas single-unit recording in the putamen has revealed that putaminal neurons are related to specific parameters of movement (DeLong and Strick, 1974; Crutcher and deLong, 1983a, b). The present study, which has shown that projections from widespread areas of association cortex extend into the anterior putamen, as well as throughout the caudate, suggests that the anterior putamen is connectionally and, perhaps, functionally more like the caudate than it is similar to the rest of the putamen. Thus, the nuclear subdivisions, caudate and putamen, may have resulted from arbitrary splitting of the neostriatum by fibers of the internal capsule. From a functional standpoint, it may be more useful to distinguish an "association neostriatum," which includes the caudate nucleus and anterior putamen, from a "motor neostriatum," which includes more posterior regions of the putamen.

Lesion studies have long suggested that there is, in addition, functional specialization within the caudate nucleus and that this functional differentiation reflects the functional specialization of anatomically connected cortex. Rosvold and his colleagues (Rosvold et al., 1958; Battig et al., 1960, 1962; Divac et al., 1967) have shown that circumscribed lesions confined centrally within the head of the caudate produced deficits similar to those observed following ablation of the DPC, whereas lesions ventrally placed in the head of the caudate resulted in deficits similar to those produced by ablation of the ORB. More recently, Rolls et al. (1983) and Caan et al. (1984) have substantiated the evidence for functional heterogeneity by showing that the activity of neurons in the head of the caudate is related to tasks that involve the ORB, whereas units in the tail are responsive during tasks that require temporal cortical activation.

The long-standing evidence for a close functional linkage between cortex and neostriatum is only now becoming fully recognized and incorporated into contemporary conceptions of the role of the striatam in normal and diseased states. Thus, recent studies have shown that damage to the neostriatum mimics the effects of pathology within afferent areas. For example, a computer tomographic reassessment of neuropathology in patients with Broca's aphasia revealed a high incidence of damage in the neostriatum and little indication of cortical injury to Broca's area (Gordon, 1984). Evidence of involvement of the caudate and putamen in aphasic disorders has also been reported by Damasio et al. (1982) and by Naeser et al. (1982). In Huntington's disease, cognitive dysfunction has often been termed "subcortical dementia" because of the prominence of neostriatal atrophy associated with the disease (Forno and Jose, 1973; Mayeux et al., 1981). Moreover, a recent study using ${ }^{18} \mathrm{~F}$ fluorodeoxyglucose and a computed tomographic scan has shown that dementia in Huntington's disease correlates with hypometabolic changes in the head of the caudate nucleus and not with cortical atrophy or reduced cortical metabolism (Kuhl et al., 1982). These neurological findings fit extremely well with the idea that a lesion in the caudate or putamen, which necessarily disconnects the cortex from its major subcortical target area, produces cognitive impairment identical to that resulting from injury to the disconnected cortex.

The present study indicates that each medial-lateral sector of the neostriatum processes a subset of associational cortical information that is distinct from that of all other sectors and therefore establishes an anatomical basis for functional mosaicism in the neostriatum. In light of this newly defined medial-lateral organization, a behavioral and physiological re-examination of the caudate and putamen with respect to localization of function in one or another of these cortically defined domains might ultimately explain the variation in symptomology that results from injury or disease afflicting selective regions of the neostriatum.

\section{References}

Baleydier, C., and F. Mauguiere (1980) The duality of the cingulate gyrus in monkey. Brain 103: 525-554.

Battig, K., H. E. Rosvold, and M. Mishkin (1960) Comparison of the effects of frontal and caudate lesions on delayed response and delayed alternation. J. Comp. Physiol. Psychol. 51: 437-444.

Battig, K., H. E. Rosvold, and M. Mishkin (1962) Comparison of the effects of frontal and caudate lesions on discrimination learning in monkeys. $J$. Comp. Physiol. Psychol. 55: 458-463.

Beckstead, R. M. (1979) An autoradiographic examination of corticocortical and subcortical projections in the medial-dorsal-projection (prefrontal) cor tex in the rat. J. Comp. Neurol. 184: 43-62.

Caan, W. D. I. Perrett, and E. T. Rolls (1984) Responses of striatal neurons in the behaving monkey. II. Visual processing in the caudal neostriatum. Brain Res. 290: 53-65.

Crutcher, M. D., and M. R. DeLong (1983a) Single cell studies of the primate putamen. I. Functional organization. Exp. Brain Res. 53: 233-243.

Crutcher, M. D., and M. R. DeLong (1983b) Single cell studies of the primate putamen. II. Relations to direction of movement and patterns of muscular activity. Exp. Brain Res. 53: 244-258.

Damasio, A. R., H. Damasio, M. Rizzo, N. Varney, and F. Gersh (1982) Aphasia with nonhemorragic lesions in the basal ganglia and internal capsule. Arch. Neurol. 39: 15-20

DeLong, M. R., and P. L. Strick (1974) Relation of basal ganglia, cerebellum and motor cortex units to ramp and ballistic limb movements. Brain Res. 71: 327-335.

Devito, J. L., and O. A. Smith, Jr. (1964) Subcortical projections of the prefrontal lobe of the monkey. J. Comp. Neurol. 123: 413-424.

DiFiglia, M., T. Pasik, and P. Pasik (1978) A Golgi study of afferent fibers in the neostriatum of monkeys. Brain Res. 152: 341-347.

Divac, J., H. E. Rosvold, and M. K. Szwarcbart (1967) Behavioral effects of selective ablation of the caudate nucleus. J. Comp. Physiol. Psychol. 63: 184-190.

Dunnett, S. B., A. Björklund, U. Stenevi, and S. D. Iversen (1981a) Grafts of embryonic substantia nigra reinnervating the ventrolateral striatum ameliorate sensorimotor impairments and akinesia in rats with 6-OHDA lesions of the nigrostriatal pathway. Brain Res. 229: 209-217.

Dunnett, S. B., A. Björklund, U. Stenevi, and S. D. Iversen (1981b) Behavioral recovery following transplantation of substantia nigra in rats subjected to 6-OHDA lesions of the nigrostriatal pathway. II. Bilateral lesions. Brain Res. 229: 459-470.

Forno, L. S., and C. Josc (1973) Huntington's chorea: A pathological study Adv. Neurol. 1: 453-470.

Goldman, P. S., and W. J. H. Nauta (1977a) Columnar distribution of corticocortical fibers in the frontal association, lirnbic, and motor cortex of the developing rhesus monkey. Brain Res. 122: 393-413.

Goldman, P. S., and W. J. H. Nauta (1977b) An intricately patterned prefrontocaudate projection in the rhesus monkey. J. Comp. Neurol. 171:369-386.

Goldman, P. S., and H. E. Rosvold (1970) Localization of function within the prefrontal cortex of the rhesus monkey. Exp. Neurol. 27: 291-304.

Goldman, P. S., H. E. Rosvold, B. Vest, and T. W. Galkin (1971) Analysis of the delayed alternation deficit produced by dorsolateral prefrontal lesions in the rhesus monkey. J. Comp. Physiol. Psychol. 77: 262-280.

Goldman-Rakic, P. S. (1981) Prenatal formation of cortical input and development of cytoarchitectonic compartments in the neostriatum of the rhesus monkey. J. Neurosci. 1: 721-735.

Goldman-Rakic, P. S. (1982) Cytoarchitectonic heterogeneity of the primate 
neostriatum: Subdivision into island and matrix cellular compartments. J. Comp. Neurol. 205: 398-413.

Goldman-Rakic, P. S., and M. L. Schwartz (1982) Interdigitation of contralateral and ipsilateral columnar projections to frontal association cortex in primates. Science 216: 755-757.

Gordon, W. P. (1985) Neuropsychological assessment of aphasia. In Speech and Language Evaluation in Neurology: Adult Disorders, J. Darby, ed. Grune and Stratton, New York, in press.

Graybiel, A. M., C. W. Ragsdale, Jr., E. S. Yoneoka, and R. P. Elde (1981) An immunohistochemical study of enkephalin and other neuropeptides in the striaturn of the cat with evidence that the opiate peptides are arranged to form mosaic patterns in register with the striosomal compartments visible by acetylcholinesterase staining. Neuroscience 6: 377-397.

Griffin, G., L. R. Watkins, and D. J. Mayer (1979) HRP pellets and slowrelease gels; two new techniques for greater localization and sensitivity. Brain Res. 168: 595-601

Groves, P. M. (1983) A theory of the functional organization of the neostriatum and the neostriatal control of voluntary movement. Brain Res. Rev. 5: 109132.

Haber, S. R, and W. J. H. Nauta (1983) Ramifications of the globus pallidus in the rat as indicated by patterns of immunohistochemistry. Neuroscience 9: 245-260

Hassler, R., J. W. Chung, U. Rinne, and A. Wagner (1978) Selective degeneration of two out of the nine types of synapses in cat caudate after cortical lesions. Exp. Brain Res. 31: 67-80.

Hubel, D. H., T. N. Wiesel, and D. M. K. Lam (1974). Autoradiographic demonstration of ocular-dominance columns in the monkey striate cortex by means of transneuronal transport. Brain Res. 79: 273-279.

Johnson, T. N., H. E. Rosvold, and M. Mishkin (1968) Projections from behaviorally defined sectors of the prefrontal cortex to the basal ganglia, septum, and diencephalon of the monkey. Exp. Neurol. 21: 20-34.

Jones, E. G., J. D. Coulter, H. Burton, and R. Porter (1977) Cells of origin and terminal distribution of corticostriatal fibers arising in the sensorymotor cortex of monkeys. J. Comp. Neurol. 173: 53-80.

Kelley, A. E., and V. B. Domesick (1982) The distribution of the projection from the hippocampal formation to the nucleus accumbens in the rat: An anterograde- and retrograde-horseradish peroxidase study. Neuroscience 7: $2321-2335$

Kelley, A. E., V. B. Domesick, and W. J. H. Nauta (1982) The amygdalostriatal projection in the rat-An anatomical study by anterograde and retrograde tracing methods. Neuroscience 7: 615-630.

Kemp, J. M., and T. P. S. Powell (1970) The cortico-striate projection in the monkey. Brain 93; 525-546.

Kemp, J. M., and T. P. S. Powell (1971) The termination of fibres from the cerebral cortex and thalamus upon dendritic spines in the caudate nucleus: A study with the Golgi method. Philos. Trans. R. Soc. Lond. (Biol.) 262: 429-439.

Kievit, J. and H. G. J. M. Kuypers (1977) Organization of the thalamo-cortical connexions to the frontal lobe in the rhesus monkey. Exp. Brain Res. 29: 299-322

Kocsis, J. D., M. Sugimoci, and S. T. Kitai (1977) Convergence of excitatory synaptic inputs to caudate spiny neurons. Brain Res. 124: 403-413.

Kuhl, D. E., M. E. Phelps, C. H. Markham, E. J. Metter, W. H. Riege, and J. Winter (1982) Cerebral metabolism and atrophy in Huntington's disease determined by ${ }^{18} \mathrm{FDG}$ and computed topographic scan. Ann. Neurol. 12: 425-434.

Künzle, H. (1975) Bilateral projections from precentral motor cortex to the putamen and other parts of the basal ganglia. An autoradiographic study in Macaca fascicularis. Brain Res. 88: 195-209.

Künzle, $H$. (1977) Projections from the primary somatosensory cortex to basal ganglia and thalamus in the monkey. Exp. Brain Res. 30: 481-492.

Künzle, H. (1978) An autoradiographic analysis of the efferent connections from premotor and adjacent prefrontal regions (areas 6 and 9) in Macaca fascicularis. Brain Behav. Evol. 15: 185-234

Leichnetz, G. R. (1980) An intrahemispheric columnar projection between two cortical multisensory convergence areas (inferior parietal lobule and prefrontal cortex): An anterograde study in macaque using HRP gel. Neurosci. Lett. 18: 119-124.

Mayeux, R., Y. Stern, J. Rosen, and D. F. Benson (1981) Subcortical dementia: A recognizable clinical entity. Trans. Am. Neurol. Assoc. 106: 313-316.

Mesulam, M. -M. (1978) Tetramethylbenzidine for horseradish peroxidase neurochemistry: A non-carcinogenic blue reaction product with superior senstivity for visualizing neural afferents and efferents. J. Histochem. Cytochem. 26: 106-117.

Mesulam, M. -M., G. W. Van Hoesen, D. N. Pandya, and N. Geschwind (1977) Limbic and sensory connections of the inferior parietal lobule (area $P G)$ in the rhesus monkey: A study with a new method for horseradish peroxidase histochemistry. Brain Res. 136: 393-414.

Naeser, M. A., M. P. Alexander, N. Helm-Estabrooks, H. L. Levine, S. A. Laughlin, and N. Geschwind (1982) Aphasia with predominantly subcortical lesion sites: Description of three capsular/putaminal aphasia syndromes. Arch. Neurol. 39: 2-14.

Niki, H., M. Sakai, and K. Kubota (1972) Delayed alternation performance and unit activity of the caudate head and medial orbito-frontal gyrus in the monkey. Brain Res. 38: 343-353

Pandya, D. N., P. Dye, and N. Butters (1971) Efferent cortico-cortical projections of the prefrontal cortex in the rhesus monkey. Brain Res. 31: 35-46.

Pandya, D. N., G. W. Van Hoesen, and M. -M. Mesulam (1981) Efferent connections of the cingulate gyrus in the rhesus monkey. Exp. Brain Res. 42: $319-330$

Parent, A., C. Bouchard, and Y. Smith (1984) The striatopallidal and striatonigral projections: Two distinct fiber systems in primate. Brain Res. 303: 385-390.

Pasik, P., T. Pasik, and M. DiFiglia (1976) Quantitative aspects of neuronal organization in the neostriatum of the macaque monkey. In The Basal Ganglia, M. D. Yahr, ed., pp. 57-90, Raven Press, New York.

Pearson, R. C. A. K. C. Gatter, and T. P. S. Powell (1983) The cortical relationships of certain basal ganglia and the cholinergic basal forebrain nuclei. Brain Res. 26: 327-330.

Petras, J. M. (1969) Some efferent connections of the superior and inferior parietal lobules with the basal ganglia, diencephalon and midbrain in the rhesus monkey. Anat. Rec. 163: 243-244.

Ragsdale, C. W., Jr., and A. M. Graybiel (1981) The fronto-striatal projection in the cat and monkey and its relationship to inhomogeneities established by acetylcholinesterase histochemistry. Brain Res. 208: 259-266.

Rakic, P. (1976) Prenatal genesis of connections subserving ocular dominance in the rhesus monkey. Nature 261: 467-471.

Rakic, P. (1981) Development of visual centers in the primate brain depends on binocular competition before birth. Science 214: 928-931.

Rolls, E. T., S. J. Thorpe, and S. P. Maddison (1983) Responses of striatal neurons in the behaving monkey. I. Head of the caudate nucleus. Behav. Brain Res. 7: 179-210.

Rosvold, H. E., M. Mishkin, and M. K. Szwarcbart (1958) Effects of subcortical lesions in monkeys on visual-discrimination and single-alternation performance. J. Comp. Physiol. Psychol. 51: 437-444.

Schwartz, M. L., and P. S. Goldman-Rakic (1982) Single cortical neurones have axon collaterals to ipsilateral and contralateral cortex in fetal and adult primates. Nature 299: 154-156.

Schwartz, M. L., and P. S. Goldman-Rakic (1984) Callosal and intrahemispheric connectivity of the prefrontal association cortex in rhesus monkey: Relation between intraparietal and principal sulcal cortex. J. Comp. Neurol. 226: 403-420.

Van Hoesen, G. W., E. H. Yeterian, and R. Lavizzo-Mourney (1981) Widespread corticostriate projections from temporal cortex of the rhesus monkey. J. Comp. Neurol. 199: 205-219.

Whitlock, D. G., and W. J. H. Nauta (1956) Subcortical projections from the temporal neocortex in Macaca mulatta. J. Comp. Neurol. 106: 182-212.

Yeterian, E. H., and G. W. Van Hoesen (1978) Cortico-striate projections in the rhesus monkey: The organization of certain cortico-caudate connections. Brain Res. 139: 43-63. 